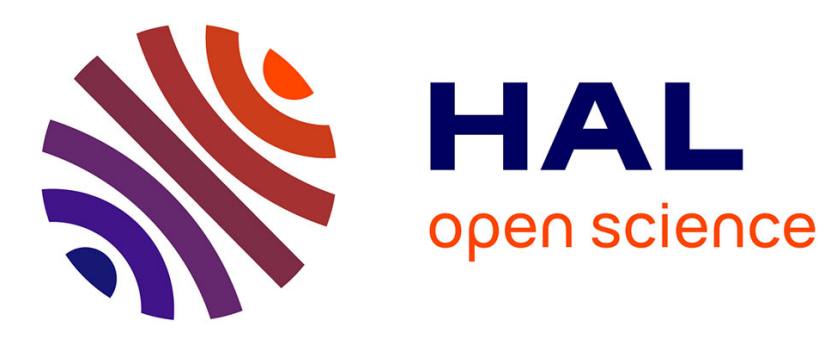

\title{
Nonlinear dynamic stability of cylindrical shells under pulsating axial loading via transient Finite Element Analysis
}

\author{
Fabio Rizzetto, Eelco Jansen, Matteo Strozzi, Francesco Pellicano
}

\section{To cite this version:}

Fabio Rizzetto, Eelco Jansen, Matteo Strozzi, Francesco Pellicano. Nonlinear dynamic stability of cylindrical shells under pulsating axial loading via transient Finite Element Analysis. Thin-Walled Structures, 2019, 143, 10.1016/j.tws.2019.106213 . hal-02263728v2

\section{HAL Id: hal-02263728 \\ https://hal.science/hal-02263728v2}

Submitted on 5 Aug 2019

HAL is a multi-disciplinary open access archive for the deposit and dissemination of scientific research documents, whether they are published or not. The documents may come from teaching and research institutions in France or abroad, or from public or private research centers.
L'archive ouverte pluridisciplinaire HAL, est destinée au dépôt et à la diffusion de documents scientifiques de niveau recherche, publiés ou non, émanant des établissements d'enseignement et de recherche français ou étrangers, des laboratoires publics ou privés. 


\title{
Nonlinear dynamic stability of cylindrical shells under pulsating axial loading via Finite Element analysis using numerical time integration
}

\author{
Fabio Rizzetto†, Eelco Jansen*, Matteo Strozzi* and Francesco Pellicano ${ }^{\circ}$
}

\author{
$\uparrow$ Ferrari SpA, \\ Via Abetone Inferiore 4, 41053 Maranello (Modena), Italy \\ fabio.rizzetto14@gmail.com \\ † Institute of Structural Analysis, Leibniz Universität Hannover, \\ Appelstrasse 9A, 30167 Hannover, Germany \\ e.jansen@isd.uni-hannover.de
}

*Department of Sciences and Methods for Engineering, University of Modena and Reggio Emilia,

Via Giovanni Amendola 2, 42122 Reggio Emilia, Italy

matteo.strozzi@unimore.it

'Department of Engineering "Enzo Ferrari”, University of Modena and Reggio Emilia, Via Vivarelli 10/1, 41125 Modena, Italy

francesco.pellicano@unimore.it

Corresponding Author

Dr. Eelco Jansen

Institute of Structural Analysis,

Leibniz Universität Hannover,

Appelstrasse 9A, 30167 Hannover, Germany

Email: e.jansen@isd.uni-hannover.de 


\begin{abstract}
Nonlinear dynamic stability investigations for isotropic and composite cylindrical shells under pulsating axial loading are carried out through Finite Element analysis using numerical time integration. In particular, important characteristics of the geometrically nonlinear behaviour are systematically studied through Finite Element analysis. The results of the Finite Element analysis are compared with results obtained in earlier studies using semi-analytical procedures. In order to facilitate the evaluation and the comparison of these two complementary approaches, a modal projection procedure has been developed for the Finite Element analysis. Critical dynamic loads and frequency-response curves for isotropic and composite shells under pulsating loading obtained with the Finite Element analysis using numerical time integration are shown to be generally in good qualitative agreement with the results of earlier semi-analytical work. The analysis of the modal amplitude achieved via the modal projection procedure also makes it possible to study the interactions between contributing modes and to observe and interpret interesting phenomena such as the occurrence of travelling waves in the circumferential direction of the shell.
\end{abstract}

\title{
Keywords
}

Nonlinear vibrations, dynamic stability, cylindrical shells 


\section{Introduction}

Thin-walled shell structures are important structural components in many branches of engineering; these structures are prone to buckling instabilities under static and dynamic compressive loading and may be directly or parametrically excited into resonance at their natural frequencies by dynamic loads. The stability of thin-walled structures under dynamic loading constitutes a relevant research area; in particular, the parametric excitation (vibration buckling under pulsating loading) represents an important topic in the dynamic stability of shell structures.

Because of the theoretical and practical significance of these structural components, the parametric excitation and nonlinear vibration problem of cylindrical shells has received considerable attention. The vibration buckling problem of shells under pulsating loading has often been investigated within a semi-analytical context. It has been recognized that essential information about the nonlinear dynamic behaviour of structures can be efficiently obtained by means of analytical-numerical, lowdimensional models, i.e. models with a small number of degrees of freedom. The essential ideas of parametric excitation for shell problems are discussed by Hsu [1]. Early investigations in the field of the parametric excitation of cylindrical shells were carried out by Yao [2-3], Vijayaraghavan and Evan-Iwanowski [4] and Nagai and Yamaki [5]. Laminated structures have been studied by Argento [6] and Argento and Scott [7-8]. Further semi-analytical work includes the work of Popov et al. [9], Gonçalves and Del Prado [10], and Dey and Ramachandra [24]. The extension of semi-analytical approaches to multi-mode analyses has been presented and used in various works by Amabili, Pellicano and their colleagues [11-15] on nonlinear vibrations and parametric excitation of cylindrical shells. A low-dimensional model with a small number of degrees of freedom, introduced earlier by Jansen [16] to capture important characteristics of the nonlinear vibration behaviour of shells, was used to analyse the dynamic stability behaviour of anisotropic cylindrical shells under dynamic axial loading [17].

The possibility to carry out a nonlinear dynamic response analysis using numerical time integration (in the literature also often denoted as "transient" analysis) for shell structures is standardly available within finite element codes. Because of their imperfection-sensitive behaviour, relevant shell configurations such as cylindrical shells have received considerable attention in dynamic buckling investigations, e.g. by Saigal et al. [20], Yaffe and Abramovich [21] and Bisagni [22]. Finite element studies based on numerical time integration is, however, computationally expensive. This in particular holds for shells under pulsating loading, which have been investigated through Finite Element Analysis, e.g. Ganapathi et al. [23], but to date have not been analysed through systematic Finite Element studies using numerical time integration. 
The present paper contains two main new contributions. For the specific problem that will be considered, the dynamic stability of cylindrical shells under parametric excitation, earlier mainly semi-analytical methods have been used. First new contribution corresponds to the application of the Finite Element method. Due to the versatility in modelling in analysis capabilities of this method, it can be used to capture also details of the characteristics of the nonlinear behaviour of the structure. The objective is to show the feasibility to carry out these full model analyses, which ask special care when numerical time integration is employed, in view of the - also from today's perspective - considerable computational resources required. The traditional semi-analytical models can typically be used only for idealized configurations and idealized conditions. Second important contribution of the present paper is the assessment of the range of validity of a specific semianalytical, low-dimensional model earlier used to investigate the essential features of the dynamic stability problem of cylindrical shells under parametric excitation.

These two new contributions will be made possible through the development of a novel modal projection procedure. This post-processing procedure to interpret the behaviour of the full model will play an important role for the investigations to be presented. The approach also has the potential to be used in a reduced order model approach. In earlier work, the Proper Orthogonal Decomposition has been presented as an appropriate tool for constructing a reduced order model for the class of problems analysed in the present paper [29]. Also the concept of "nonlinear modes" has been used for this purpose [30, 31]. A Finite Element integrated perturbation-type approach has been used as a reduction method to study the nonlinear (large amplitude) vibration behaviour and dynamic buckling behaviour of composite cylindrical shells under axial step loading [18, 19].

In this paper, the large amplitude vibration behaviour of both isotropic and composite cylindrical shells under pulsating loading is investigated by using transient Finite Element analysis using numerical time integration in order to obtain accurate solutions. The Finite Element analysis allows an accurate satisfaction of idealized or realistic boundary conditions, makes it possible to include all relevant inertial effects, and enables capturing the complicated interaction between the "trivial", axisymmetric fundamental dynamic state and the parametrically excited "nontrivial", asymmetric dynamic state. Key issues for the numerical analysis will be addressed. A Finite Element modal projection procedure will be developed, which facilitates the interpretations of the Finite Element analysis using numerical time integration, in particular the comparison between Finite Element calculations and the results of semi-analytical approaches available in the literature. 


\section{Problem formulation on the basis of a semi-analytical approach}

In the present section, the problem of parametric excitation of a composite cylindrical shell under pulsating axial loading is described on the basis of the governing equations and displacement functions for this problem following the formulation used in Ref. [17].

\subsection{Governing equations}

The equations governing the nonlinear dynamic behaviour of a cylindrical shell are presented. The shell is loaded by an axial compressive force $P$ and an external pressure $p$ which can both depend on time. It is assumed that the radial displacement $w$ is positive inward (see Figure 1). Applying the usual membrane stress resultants $N_{x}, N_{y}$ and $N_{x y}$, and introducing the Airy stress function $F$ defined by $N_{x}=F_{, y y}, N_{y}=F_{, x x}$ and $N_{x y}=-F_{, x y}$, the Donnell-type nonlinear imperfect shallow shell equations for an anisotropic material can be written as

$$
\begin{aligned}
& L_{A^{*}}(F)-L_{B^{*}}(w)=-\frac{1}{R} w_{, x x}-\frac{1}{2} L_{N L}(w, w+2 \bar{w}) \\
& L_{B^{*}}(F)-L_{D^{*}}(w)=\frac{1}{R} F_{x x}+L_{N L}(F, w+\bar{w})+p-\bar{\rho} h w, t t
\end{aligned}
$$

where the variables $w$ and $F$ depend on the spatial coordinates $x, y$ and time $t, R$ is the shell radius, $\bar{w}$ denotes a stress-free radial initial imperfection, $\bar{\rho} h w_{t t}$ is the radial inertia term, $\bar{\rho}$ is the averaged specific mass of the laminate, $h$ is the reference shell thickness.

The $4^{\text {th }}$ order linear differential operators

$$
\begin{aligned}
L_{A^{*}}(\cdot)= & A_{22}^{*}(\cdot)_{x x x x}-2 A_{26}^{*}(\cdot)_{x x x y}+\left(2 A_{12}^{*}+A_{66}^{*}\right)(\cdot)_{x x y y}-2 A_{16}^{*}(\cdot)_{x y y y}+A_{11}^{*}(\cdot)_{, y y y y} \\
L_{B^{*}}(\cdot)= & B_{21}^{*}(\cdot)_{x x x x}+\left(2 B_{26}^{*}-B_{61}^{*}\right)(\cdot)_{x x x y}+\left(B_{11}^{*}+B_{22}^{*}-2 B_{66}^{*}\right)(\cdot)_{x x y y} \\
& +\left(2 B_{16}^{*}-B_{62}^{*}\right)(\cdot)_{x y y y}+B_{12}^{*}(\cdot)_{y y y y} \\
L_{D^{*}}(\cdot)= & D_{11}^{*}(\cdot)_{x x x x}+4 D_{16}^{*}(\cdot)_{x x x y}+2\left(D_{12}^{*}+2 D_{66}^{*}\right)(\cdot)_{x x y y}+4 D_{26}^{*}(\cdot)_{x y y y}+D_{22}^{*}(\cdot)_{, y y y y}
\end{aligned}
$$

depend on the stiffness properties of the laminate, the elements of the partially inverted ABDmatrix [17], and the nonlinear operator defined by

$$
L_{N L}(S, T)=S_{, x x} T_{, y y}-2 S_{, x y} T,_{x y}+S_{, y y} T_{, x x}
$$

reflects the geometric nonlinearity. 
In particular, Eq. (1) guarantees the compatibility of the strains and the radial displacement field while Eq. (2) is the equation of motion (dynamic equilibrium equation) in radial direction.

The first step for solving the equations is to transform them into a finite set of nonlinear ordinary differential equations (ODEs) using the Galerkin method, through which we can describe the deformation using a finite number of degrees of freedom (DOFs).

The radial displacement $w(x, \theta, t)$ can be written as

$$
w(x, \theta, t)=\sum_{J=1}^{N} a_{j}(t) \psi_{j}(x, \theta)
$$

The coefficients $\mathrm{a}_{\mathrm{j}}(\mathrm{t})$ are now the DOFs of the problem. After suitably expanding the Airy function and projecting the governing equations using the Galerkin procedure, one can obtain the following set of ODEs,

$$
\ddot{a}_{j}+2 \zeta_{j} \omega_{j} \dot{a}_{j}+\sum_{i=1}^{N} z_{j, i} a_{i}+\sum_{i=1}^{N} \sum_{i=1}^{N} z_{j, i} a_{i} a_{i}+\sum_{i=1}^{N} \sum_{i=1}^{N} \sum_{k=1}^{N} z_{j, i, k} a_{i} a_{j} a_{k}=g(t)
$$

\subsection{Displacement functions}

The choice of the trial functions used for the radial displacement $w$ is crucial in order to obtain good quality results in the case of nonlinear vibrations; studies on convergence in the nonlinear case are reported in Refs. [12-15]. It is important to include axisymmetric modes in the expression for the radial displacement.

Let us consider a circular cylindrical shell with classical, simply supported boundary conditions (SS-3 boundary conditions), excited by a radial harmonic load with circular frequency $\Omega$ to vibrate in the resonant condition of the mode $(\mathrm{m}, \mathrm{n})$, where $m$ is the number of longitudinal half-waves and $n$ the number of circumferential full waves. It is important to include the following modes in the displacement function assumed for the radial displacement [11, 12]:

- resonant mode, including both driven and companion modes

- asymmetric modes with $k \times n$ circumferential full waves, where $k$ is an integer

- axisymmetric modes 
In certain cases, additional modes with natural frequency close to the ratio 1:1, 1:2, 1:3 with respect to the frequency of excitation, which in general may have an influence in the response, should be included. In the numerical calculations in Ref. [12], the following 23-DOFs expansion was assumed:

$$
\begin{gathered}
w(x, \theta, t)= \\
\sum_{m=1}^{3} \sum_{k=1}^{3}\left[A_{m, n}(t) \cos (k n \vartheta)+B_{m, n}(t) \sin (k n \theta)\right] \sin \left(\lambda_{m} x\right)+ \\
\sum_{m=1}^{5} A_{m, 0}(t) \sin \left(\lambda_{2 m-1} x\right)
\end{gathered}
$$

In the expansion presented in Ref. [17], the most essential modes were retained and the number of DOFs was thereby reduced to four. Driven and companion resonant modes and two specific axisymmetric modes were included. One of the axisymmetric modes satisfies a crucial coupling condition with the driven and companion mode. In the numerical calculations the expansion used in Ref. [17] is

$w(x, \theta, t)=C_{0}(t)+C_{1}(t) \cos \left(\frac{2 m \pi x}{L}\right)+A(t) \sin \left(m \pi \frac{x}{L}\right) \cos \left(n \theta-\frac{\tau_{R}}{R} x\right)+B(t) \sin \left(m \pi \frac{x}{L}\right) \cos \left(n \theta-\frac{\tau_{R}}{R} x\right)$

where $\tau_{R}$ is Khot's skewedness parameter, which is equal to zero in the case of an isotropic shell but can reflect the possible skewedness of the deflection pattern of an anisotropic shell. In the case of skewed modes and for large amplitude deflections, the expression satisfies the classical boundary condition SS-3 only approximately. 


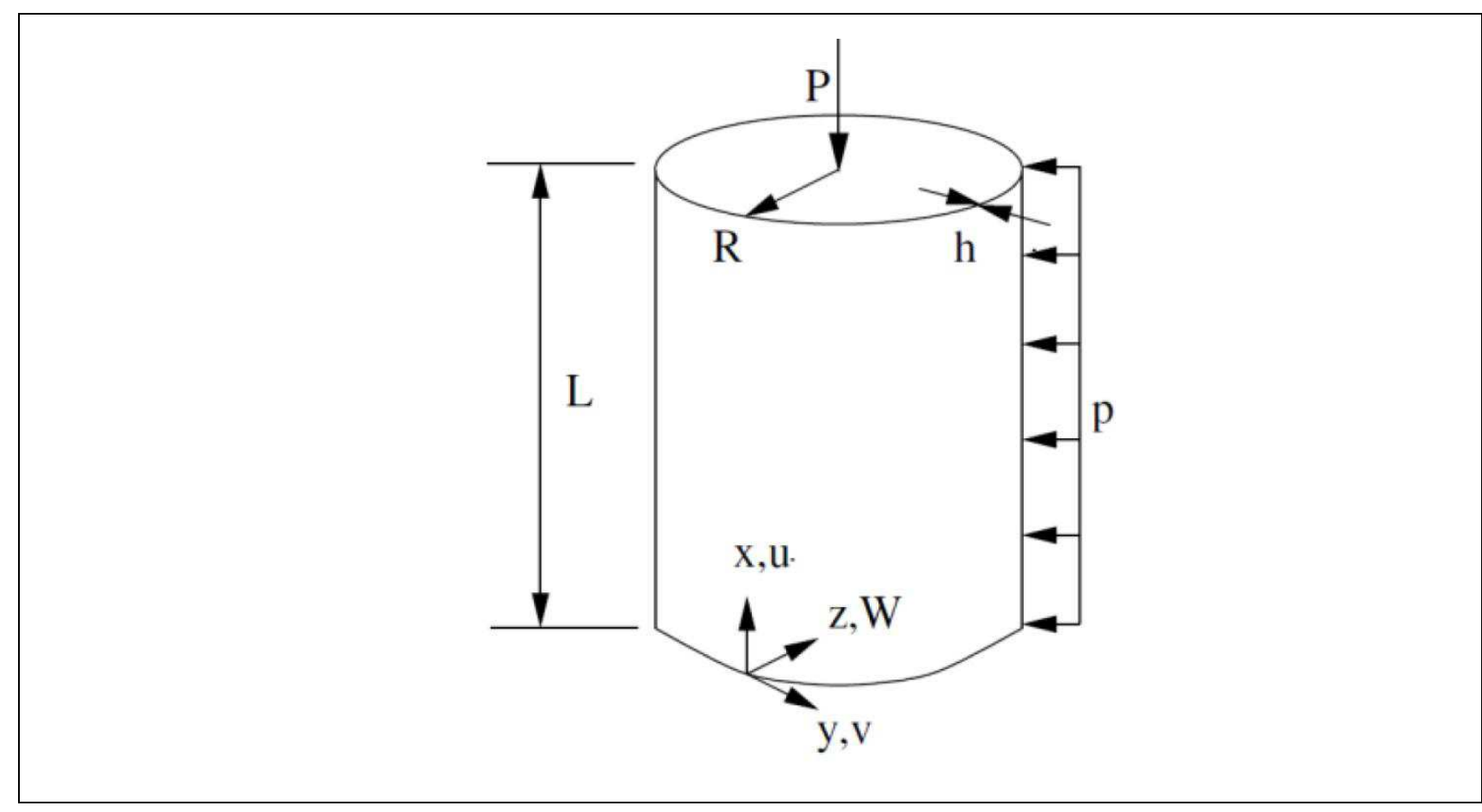

Figure 1. Shell geometry, coordinate system and applied loading within semi-analytical nonlinear dynamic analysis of composite cylindrical shells [17].

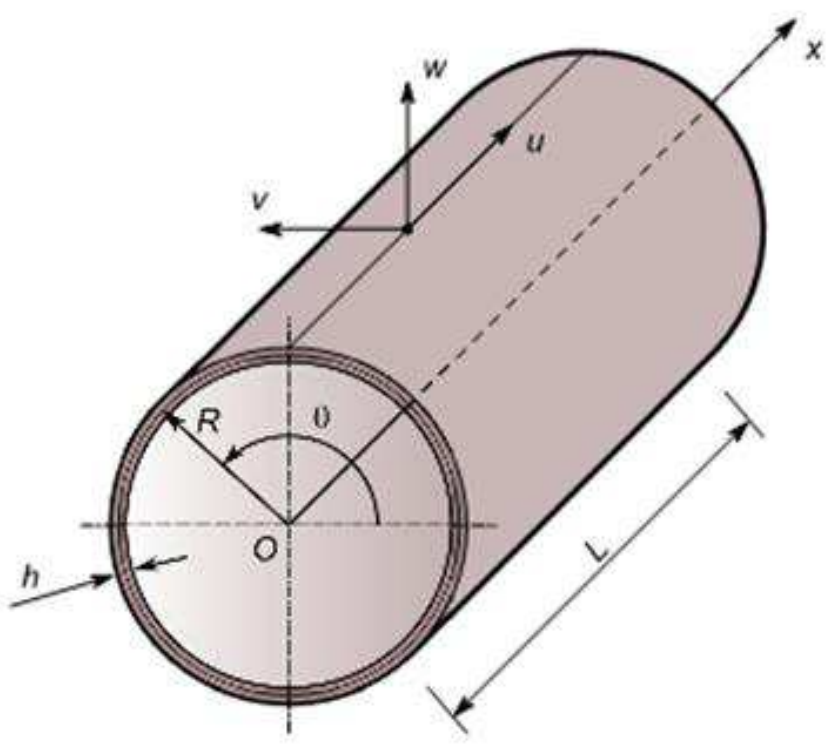

Figure 2. Shell geometry and coordinate system for Finite Element analysis. 


\section{Finite Element Analysis}

\subsection{Nonlinear Dynamic Analysis}

The nonlinear dynamics simulations were performed using the Finite Element (FE) software Abaqus 2016 [25]. A four-node general-purpose shell element with reduced integration and finite membrane strains (the Abaqus S4R shell element) was employed. The time integration scheme used is the implicit HHT (Hilbert-Hughes-Taylor) method. This integration scheme is unconditionally stable for linear problems and has been shown to give appropriate stability properties also for certain nonlinear problems, but it introduces a certain amount of numerical damping in the system. In order to reduce the influence of this numerical damping, one must carefully set the parameters of the method in an approach in which small time increments are used to accurately represent the vibrational response of the structure while keeping the numerical energy dissipation at a minimum ("Transient Fidelity" option in Abaqus, see Table 1).

Regarding the time step of the integration, an automatic scheme of variable time step available in Abaqus is used. Three parameters are defined within this approach: initial suggested time step, minimum time step and maximum time step. After a convergence analysis, the initial and maximum time steps were always chosen smaller than 1/80 $T$, where $T$ is the time period of the harmonic forcing; the integrator is free to use a smaller time step if needed. Using a low or negligible damping, the influence of the time step choice is crucial and one should apply a small time step in order to reduce the deviation of the solution.

\subsection{Modal projection procedure}

In order to simplify the interpretation of the dynamic response of the structure, an innovative postprocessing procedure was developed. The deflection of the cylindrical shell can be described using a time depending vector $\overline{\mathrm{u}}(t)$. The goal is then to obtain the best approximation of this vector using a reduced basis constituted of linearly independent (in general not orthogonal) vectors. Applying this approach to the Finite Element analysis results, for each time step of the time history output, a vector $\overline{\mathrm{u}}$ with components $u_{i}$, the radial displacement associated with the node of the shell surface with coordinates $\left(\theta_{i}, R, x_{i}\right)$, can be easily assembled (considering only the radial displacement instead of all 6 DOFs at the node allows reducing the size of the problem considerably). The same procedure is used when assembling time independent vectors $\bar{\psi}_{\mathrm{i}}$ corresponding to the modal shapes obtained via linear frequency analysis. These vectors, as linear independent vectors, are appropriate candidates for building the reduced basis $\mathrm{X}$ : 


$$
X=\left[\begin{array}{llll} 
& & & \\
\bar{\Psi}_{1} & \ldots & \ldots & \bar{\psi}_{\mathrm{N}}
\end{array}\right]
$$

where $\bar{\psi}_{\mathrm{i}}$ is corresponds to a modal shape with "displacement normalization". This means that, once a vector $\bar{\psi}^{*}$ has been imported, $\bar{\psi}$ is defined as $\bar{\psi}=\frac{\bar{\psi}^{*}}{\max \psi_{\mathrm{i}}^{*}}$. For the actual deflection un, its approximation can be written in terms of reduced basis vectors,

$$
\overline{\mathrm{u}} \cong \tilde{\mathrm{u}}=\sum_{\mathrm{j}=1}^{\mathrm{N}} \mathrm{a}_{\mathrm{j}} \bar{\psi}_{\mathrm{j}}=X \overline{\mathrm{a}}
$$

where $a_{j}$ is time-dependent. In order to find the linear combination that gives the best fit of the real surface, one can minimize the norm of the error vector

$$
\|\Delta \overline{\mathrm{u}}\|^{2}=\|\overline{\mathrm{u}}-U \overline{\mathrm{a}}\|^{2}=\overline{\mathrm{u}}^{2}-\overline{\mathrm{u}} X \overline{\mathrm{a}}-X \overline{\mathrm{a}} \overline{\mathrm{u}}-X \overline{\mathrm{a}} \mathrm{X} \overline{\mathrm{a}}
$$

Imposing the gradient equal to be equal to zero, the following is obtained:

$$
\frac{d}{d \bar{a}}\left(\bar{u}^{2}-\bar{a}^{T} X^{T} \bar{u}-\left(\bar{a}^{T} X^{T}\right) \bar{u}+\bar{a}^{T} X^{T} X \bar{a}\right)=\frac{d}{d \bar{a}}\left(\bar{u}^{2}-2 \bar{a}^{T} X^{T} \bar{u}+\bar{a}^{T} X^{T} X \bar{a}\right)
$$

which leads to

$$
2 \bar{u}^{\mathrm{T}} \mathrm{X}+2 \overline{\mathrm{a}}^{\mathrm{T}} \mathrm{X}^{\mathrm{T}} \mathrm{X}=\mathrm{X}^{\mathrm{T}} \overline{\mathrm{u}}+\mathrm{X}^{\mathrm{T}} \mathrm{X} \overline{\mathrm{a}}=0
$$

Defining the matrix $S=X^{\mathrm{T}} \mathrm{X}, \overline{\mathrm{a}}$ can be determined from

$$
\mathrm{S}^{-1} \mathrm{X}^{\mathrm{T}} \overline{\mathrm{u}}=\overline{\mathrm{a}}
$$

One can demonstrate that this corresponds to a minimum by evaluating the Hessian matrix,

$$
\frac{d}{d \bar{a}}\left(X^{T} \bar{u}+X^{T} X \bar{a}\right)=X^{T} X
$$

Since $\mathrm{X}$ has maximum rank, the Hessian matrix has only positive eigenvalues. The approximation error can be monitored, for instance by evaluating the following dimensionless form, 


$$
\varepsilon=\|\Delta \overline{\mathrm{u}}\| /\|\overline{\mathrm{u}}\|
$$

where $\Delta \overline{\mathrm{u}}=\overline{\mathrm{u}}-\mathrm{X} \overline{\mathrm{a}}$. For the results presented in the following, a reasonable tolerance of less than $10 \%$ was specified for this dimensionless error. For assessing the quality of the projection this dimensionless error is compared with respect to the relative magnitude of the response $\|\overline{\mathrm{u}}\| /\left\|\overline{\mathrm{u}}_{\max }\right\|$, where $\overline{\mathrm{u}}_{\max }$ corresponds to the maximum norm of $\overline{\mathrm{u}}$.

\begin{tabular}{cll}
\hline Parameter & $\begin{array}{l}\text { Transient } \\
\text { fidelity }\end{array}$ & $\begin{array}{l}\text { Moderate } \\
\text { dissipation }\end{array}$ \\
\hline $\boldsymbol{\alpha}$ & -0.05 & -0.41421 \\
\hline $\boldsymbol{\beta}$ & 0.275625 & 0.5 \\
\hline $\boldsymbol{\gamma}$ & 0.55 & 0.911421 \\
\hline
\end{tabular}

Table 1. HHT default parameters.

\begin{tabular}{ll}
\hline & Bogdanovich's isotropic shell \\
\hline $\mathrm{L}$ & $2 \mathrm{~m}$ \\
\hline $\mathrm{R}$ & $1 \mathrm{~m}$ \\
\hline $\mathrm{h}$ & $0.01 \mathrm{~m}$ \\
\hline $\mathrm{E}$ & $4 \times 10^{10} \mathrm{~N} / \mathrm{m}^{2}$ \\
\hline$\rho$ & $2500 \mathrm{~kg} / \mathrm{m}^{3}$ \\
\hline$v$ & 0.3
\end{tabular}

Table 2. Bogdanovich's isotropic shell data.

\begin{tabular}{ll}
\hline & Popov's isotropic shell \\
\hline $\mathrm{L}$ & $0.4 \mathrm{~m}$ \\
\hline $\mathrm{R}$ & $0.2 \mathrm{~m}$ \\
\hline $\mathrm{h}$ & $2 \times 10^{-3} \mathrm{~m}$ \\
\hline $\mathrm{E}$ & $2.1 \times 10^{11} \mathrm{~N} / \mathrm{m}^{2}$ \\
\hline$\rho$ & $7850 \mathrm{~kg} / \mathrm{m}^{3}$ \\
\hline$v$ & 0.3 \\
\hline
\end{tabular}

Table 3. Popov's isotropic shell data. 


\begin{tabular}{|c|c|c|c|}
\hline \multicolumn{4}{|c|}{ Booton's anisotropic shell } \\
\hline \multirow[t]{2}{*}{ Dimensions } & & & $R=0.0678 \mathrm{~m}$ \\
\hline & & & $L=0.0959 \mathrm{~m}$ \\
\hline \multicolumn{4}{|l|}{ Laminate properties } \\
\hline \multirow[t]{5}{*}{ Number of layers: } & & & 3 \\
\hline & LAYER & angle & Thickness \\
\hline & 1 outward & $+30^{\circ}$ & $0.226 \mathrm{~mm}$ \\
\hline & 2 middle & $0^{\circ}$ & $0.226 \mathrm{~mm}$ \\
\hline & 3 inward & $-30^{\circ}$ & $0.226 \mathrm{~mm}$ \\
\hline \multicolumn{4}{|c|}{ Single layer properties } \\
\hline Material & \multicolumn{3}{|c|}{ Glass-Epoxy } \\
\hline \multirow[t]{4}{*}{ Elastic properties } & \multicolumn{3}{|c|}{$E_{11}=4.0210^{4} \mathrm{MPa}$} \\
\hline & \multicolumn{3}{|c|}{$E_{22}=1.6710^{4} \mathrm{MPa}$} \\
\hline & \multicolumn{3}{|r|}{$v_{12}=0.363$} \\
\hline & \multicolumn{3}{|c|}{$G_{12}=4.6110^{3} \mathrm{MPa}$} \\
\hline Density & \multicolumn{3}{|c|}{$\rho=2778.62 \mathrm{~kg} / \mathrm{m}^{3}$} \\
\hline
\end{tabular}

Table 4. Booton's anisotropic shell data.

\begin{tabular}{llll}
\hline \multicolumn{4}{c}{ Boundary conditions } \\
\hline Bottom edge nodes: & \multicolumn{2}{c}{$\mathrm{v}=\mathrm{w}=0$} \\
\hline Symmetry edge nodes: & \multicolumn{3}{c}{$\mathrm{u}=R_{\theta}=R_{\rho}=0$} \\
\hline & Mesh (uniform) \\
\hline & Booton & Popov & Bogdanovich \\
\hline Element type & S4R & S4R & S4R \\
\hline Element size & $0.0015 \mathrm{~m}$ & $0.004 \mathrm{~m}$ & $0.02 \mathrm{~m}$ \\
& $\times$ & $\times$ & $\times$ \\
& $0.0032 \mathrm{~m}$ & $0.02 \mathrm{~m}$ & $0.1 \mathrm{~m}$ \\
\hline Total number elements & 8520 & 3140 & 3140 \\
\hline
\end{tabular}

Table 5. Boundary conditions for shells used in the Finite Element analysis. 


\section{Numerical results}

For the numerical calculations for shells under axial pulsating loading, examples from literature of circular cylindrical shell are considered. For the case of isotropic shells, Popov's shell [26] and Bogdanovich's [27] shell are considered. For the anisotropic shells, the static and dynamic behaviour of Booton's shell [28] is considered. In Tables 2-4 the mechanical properties of these three shells are reported. In Figure 2 the coordinate system and conventions for the FE analyses are shown. In Table 5, the properties of the boundary conditions in the FE modelling are reported.

The terms "driven mode" and "companion mode" introduced in earlier studies of nonlinear vibrations of laterally excited cylindrical shells will also be used in the following analysis of a cylindrical shell under axial parametric excitation. The term "driven mode" refers to the mode that is triggered through a corresponding imperfection or initial displacement. The term driven mode is also used in those cases, in which the response mode is not fully affine to the triggering imperfection, but corresponds to a mode that is spatially to a considerable extent triggered by the imperfection applied.

For these shells the nonlinear "single mode" response behaviour is studied, comparing the basic characteristics of the nonlinear behaviour (nonlinear amplitude - frequency curves, corresponding jump phenomena, and also snap-through phenomena) with results of earlier investigations using a simplified approach. Also the two-mode "driven mode -companion mode" coupled response that has been identified as an essential characteristic for the nonlinear dynamic behaviour of cylindrical shells will be investigated. It can be shown, that the nonlinear modes corresponding to the specific, "linear" symmetric (with respect to the shell mid-length) mode considered for isotropic shells in the current work (corresponding to a mode with one half wave in the axial direction), are also symmetric with respect to the shell mid-length. Since this specific behaviour will be studied in this paper, for Bogdanovich's shell and Popov's shell, a symmetry condition will be used at the shell mid-length. It should be kept in mind that when considering the nonlinear response of the (fulllength) shell, in case a mode that is non-symmetric with respect to the shell mid-length is triggered, this potentially - through a nonlinear coupling - can result in a nonlinear response that is not symmetric with respect to the shell mid-length.

\subsection{Nonlinear dynamic response of reference shells under pulsating axial loading}

Main response characteristics will be illustrated for Bogdanovich's isotropic shell and Booton's anisotropic shell. 


\section{Bogdanovich's isotropic shell}

The first case study is the dynamic response of Bogdanovich's isotropic shell under axial pulsating load. The shell considered is simply supported (SS-3), while a uniform membrane stress resultant on the edge is specified,

$$
\widehat{N}_{x}=-\widehat{N}_{0} \cos (\Omega t)
$$

In addition, a static axial pre-load is applied through the uniform membrane stress resultant $\widetilde{N}_{x}$. The amplitudes of the dynamic and static excitations are normalized using the classical critical load $N_{c l}=\frac{E h^{2}}{R \sqrt{3\left(1-v^{2}\right)}}$, corresponding to an axial force $N_{c l}=\frac{E h^{2}}{R \sqrt{3\left(1-v^{2}\right)}} \cdot 2 \pi R$ equal to $1.5211 \times 10^{7} \mathrm{~N}$ using the data presented in Table 2.

In the calculations, the following axial load amplitudes are considered: for the dynamic load $\widehat{N}_{0} / N_{c l}=\hat{\lambda}=0.35$ and for the static pre-load $\tilde{\lambda}=\widetilde{N}_{x} / N_{c l}=0,0.1,0.25,0.5$.

A single mode imperfection is also considered

$$
\bar{w}=0.01 \cdot h \cdot \sin \left(\pi \frac{x}{L}\right) \cdot \cos (5 \theta)
$$

and the damping is neglected.

In [17] the following 3 DOF expansion was used

$$
w=C_{0}(t)+C_{1}(t) \cos \left(\frac{2 \pi x}{L}\right)+A(t) \sin \left(\pi \frac{x}{L}\right) \cos (5 \theta)
$$

In the FE analysis, the radial displacement $w$ is considered positive outward. Damping is neglected. Homogeneous initial conditions are applied, corresponding to the initial conditions in the semianalytical analysis in Ref. [17]: $A(0)=\dot{A}(0)=C_{0}(t)=\dot{C}_{0}(t)=C_{1}(t)=\dot{C}_{1}(t)=0$.

In order to analyse the linear and the nonlinear behaviour corresponding to the principal MathieuHill parametric instability, the shell is excited at frequency $\Omega$ close to $2 \omega_{\operatorname{lin}}$, where $\omega_{\text {lin }}$ is the linear (small amplitude) frequency of the imperfect and preloaded shell. In order to obtain the frequencyload curves for specified load amplitudes, the governing equations are integrated in a time interval equal to 50 forcing periods $T$ and the maximum of the system response during this time interval is recorded. 
Finite Element analysis details of the model boundary conditions are listed in Table 5. It is to be noted that symmetry conditions have been used in the FE model; in particular, a symmetry plane located at $\mathrm{x}=\frac{\mathrm{L}}{2}$ and perpendicular to the axis of the cylinder is considered. This choice has the aim to reduce the computational cost and allows us to model exactly the boundary conditions SS-3. In this case a response that is symmetric with respect to the shell is studied. The load is applied with concentrated forces on the nodes of the shell edge. Single dynamic simulations for different frequency of excitation and preload levels are carried out. Referring to Eq. (12), where $\overline{\psi_{\mathrm{i}}}$ are the modes selected for the response projection, the modal projection procedure developed considers the first 100 modes of the FE linear frequency analysis and the axisymmetric modes $(1,0),(3,0)$ and $(5,0)$ as $\overline{\psi_{i}}$.

In Figure 3 the modal amplitude $a_{i}(t)$, referring again to Eq. (12), is presented for modes $(1,0)$, $(3,0),(5,0)$ and $(1,5),(1,6)$. Both the "driven" and "companion" mode are considered. Besides the strong response of modes $(1,5)$ and $(1,6)$, the inward vibration of the mode $(1,0)$ should be noted, confirming earlier observations in the literature about modes contributing in the nonlinear behaviour and more specifically the relevance of axisymmetric modes [12, 17]. Also the phase shift of $k \pi$ between "driven" and "companion" mode should be noted, where $k$ is an integer. In an earlier stage of the time history, when the parametric resonance has not yet manifested itself, the amplitude of the response is relatively small. It can be seen that the approximation error $\varepsilon=\|\Delta \overline{\mathrm{u}}\| /\|\overline{\mathrm{u}}\|$ remains modest for the larger response amplitudes occurring in a later stage of the time history (i.e. when the relative magnitude of the response $\|\overline{\mathrm{u}}\| /\left\|\overline{\mathrm{u}}_{\max }\right\|$ is large), which corresponds to the situation of interest. The maxima of the modal amplitudes frequency-response curve are presented in Figures 4 and 5. Results when ordering the modes with respect to the maximum amplitude reached during the integration time are presented in Table 6 . Considering specifically the resonant mode $(1,5)$ the curves presented in Figure 6 were obtained. The FE analysis shows a qualitatively good agreement with the semi-analytical results using only a small number of degrees of freedom shown in [17]. The softening behaviour of the response curves and the jump phenomena corresponding to the softening branch, found earlier in the semi-analytical analysis, are also observed in the present Finite Element analysis. The snap to a remote configuration, using the semi-analytical model presented in [17] seen for a preload of $0.5 \mathrm{~N}_{\mathrm{cl}}$, in the present Finite Element study already occurs for a preload of $0.25 \mathrm{~N}_{\mathrm{cl}}$. Additional studies have been carried out using the semi-analytical model of Ref. [17] (Figure 7). These calculations show, that for a number of forcing periods of 50 (Fig 7a), the snap occurs at a pre-load level around $0.30 \mathrm{~N}_{\mathrm{cl}}$. Moreover, calculations were carried out for a higher number of forcing periods. These calculations (Fig 7b) show, that in this case also with the 
semi-analytical model, for a pre-load level of $0.25 \mathrm{~N}_{\mathrm{cl}}$ a snap to a remote configuration is predicted.

An interesting phenomenon relates to the relevant modes involved in the response. In the "waterfall" charts of Figure 8, the maximum absolute value of the response $a_{i}(t)$, defined as $A_{\max }=\left|\max \left(a_{i}(t)\right)\right|$, is shown as a function of the excitation frequency (on the $\mathrm{y}$-axis) and the projected mode (along the $\mathrm{x}$-axis). The present results are in agreement with expectations $[11,12]$. In particular, a strong response of the following modes can be observed:

- both driven and companion modes

- asymmetric modes with $k \times n$ circumferential waves, where $k$ is an integer

- $\quad$ axisymmetric modes

\section{Booton's anisotropic shell}

A parametric excitation analysis was also performed for the simply supported Booton's anisotropic cylindrical shell. In this case, the simulations were made without static pre-load. The dynamic axial load is imposed through the corresponding uniform membrane stress resultant

$$
\widehat{N}_{x}=-\widehat{N}_{0} \cos (\Omega t)
$$

where $\widehat{\mathrm{N}}_{0} / \mathrm{N}_{\mathrm{cl}}=\hat{\lambda}=0.1$ and the classical buckling stress resultant $\mathrm{N}_{\mathrm{cl}}$ for a laminated shell is defined as

$$
N_{c l}=\frac{E_{11} h^{2}}{R \sqrt{3\left(1-v_{12}^{2}\right)}}
$$

in which material properties of a layer of the laminate are used (see Table 4). The three DOFs expansion used in Ref. [17] is

$$
\frac{w}{h}=C_{0}(t)+C_{1}(t) \cos \left(\frac{2 \pi x}{L}\right)+A(t) \sin \left(\pi \frac{x}{L}\right) \cos \left(6 \theta-\frac{\tau_{R}}{R} x\right)
$$

where the skewedness parameter $\tau_{R}$ imposed in [17] is equal to 0.002 , which corresponds to a very small skewedness. The skewedness of the pattern that can be extracted from the results of the Finite Element linear frequency analysis is higher (around 0.03), but can still be considered to be small. In the semi-analytical analysis in Ref. [17], a two-mode imperfection is used: 


$$
\frac{\bar{w}}{h}=0.04 \cos \left(\frac{2 \pi x}{L}\right)-0.05 \sin \left(\pi \frac{x}{L}\right) \cos \left(6 \theta-\frac{\tau_{R}}{R} x\right)
$$

Because of the anisotropy of the shell, the shell response will not be symmetric with respect to the shell mid-length and for this reason the complete circular shell is modelled. In order to model the SS-3 boundary condition while avoiding unconstrained displacements at the shell edges in the axial direction, the bottom edge of shell was connected with a support by a set of soft springs. To represent an imperfect geometry corresponding to the imperfection shape used in the semianalytical approach, the Finite Element mesh node positions were defined via a MATLAB script. Also in these simulations damping is neglected. The maximum of the modal response was considered within the first 200 forcing periods $T$ of the integration period. In the FE dynamic simulations the upper limit of the time step size was set equal to 1/160 T. In Figures 9 and 10 the results of the modal projection procedure are presented. The method makes it possible to identify the different modes involved in the response. The FE analysis shows a qualitatively good agreement with the semi-analytical results reported in Ref. [17].

\subsection{Critical dynamic load evaluation}

The present analysis is concerned with the evaluation of the critical dynamic load of a shell under axial pulsating loading. When the main parameters of the analysis case (such as the frequency of excitation) have been fixed, the stability properties of the system can be studied as a function of the load amplitude. The critical dynamic load (CDL) is defined as the load at which for small perturbations in an "asymmetric" mode, a growing solution of the asymmetric response, or a jump to a remote branch, occurs.

This evaluation of the critical dynamic load will be carried out for a simply supported isotropic shell, Popov's shell. The case of Popov's shell was earlier studied within a semi-analytical context in Ref. [12], where results were obtained via continuation methods, and in Ref. [17], via numerical time integration. Also in the present FE analysis, numerical time integration will be used. The shell is subjected to a harmonic excitation in which the frequency $\Omega$ is constant and close to $2 \omega_{\text {lin }}$ and the amplitude is increased stepwise with an increment of $0.001 \mathrm{~N}_{\mathrm{cl}}$. The duration of each step is $1000 T$, where $T$ is the forcing period. In order to interrogate the stability of the dynamic response, an initial condition for the displacement is imposed. In particular, the following initial condition for the radial displacement at time $t=0$ is applied: 


$$
w_{t=0}(\theta, x)=0.01 h \cos (5 \theta) \sin \left(\frac{\pi}{L} x\right)
$$

and the consequence of this disturbance on the response of the shell is monitored. The expansion used in [17] is Eq. (10), with $m=1, n=5\left(\tau_{R}=0\right)$. In Refs. [12] and [17] modal damping is introduced. In the current FE analysis a corresponding Rayleigh damping with parameters reported in Table 7, is used. Also in the present analysis half cylinder is modelled, so that a response symmetric with respect to the shell mid-length can be captured. In Figure 11 the time history of the radial displacement of a node located on the peak of the resonant deformed shape $w_{\text {ref }}$ is represented. It is noted that in the procedure of stepwise increasing the amplitude of the harmonic excitation, the critical load obtained (i.e. the load at which a growing solution of the asymmetric response occurs) can in certain cases depend on the starting amplitude for the analysis in combination with the initial conditions applied. Results are presented in Table 8. The modal projection procedure developed reveals the modes involved in the response, see Figure 12.

\subsection{Travelling wave investigation}

The nonlinear coupling between driven and companion mode may lead to the phenomenon of travelling waves in the circumferential direction of the shell. Considering the relevant terms related to the driven mode and companion mode in the expression for the radial deflection in Eq. (9), assuming that the time-dependent amplitudes $A_{1, n}$ and $B_{1, n}$ can be expressed as

$$
A_{1, n}(t)=a_{1, n} \cos (\omega t), \quad B_{1, n}(t)=b_{1, n} \cos \left(\omega t+\phi_{2}\right)
$$

the radial response may be written as

$$
w(x, \theta, t)=\left[a_{1, n} \cos (\omega t) \cos (n \theta)+b_{1, n} \cos \left(\omega t+\phi_{2}\right) \sin (n \theta)\right] \sin \left(\frac{\pi x}{L}\right)
$$

If $\phi_{2}=k \pi$ with $k=0,1,2, \ldots$ the response is a pure standing wave

$$
w(x, \theta, t)=\left[\left(a_{1, n} \cos (n \theta) \pm b_{1, n} \sin (n \theta)\right) \cos (\omega t)\right] \sin \left(\frac{\pi x}{L}\right)
$$

whereas if $\phi_{2} \neq k \pi$ the response can be written as 


$$
w(x, \theta, t)=\left\{\left[a_{1, n} \cos (\omega t)+b_{1, n} \sin \left(\omega t+\phi_{2}\right)\right] \cos (n \theta)+b_{1, n} \sin \left(n \theta-\omega t-\phi_{2}\right)\right\} \sin \left(\frac{\pi x}{L}\right)
$$

which is the superposition of a standing wave and a travelling wave, that becomes a pure traveling wave if $a_{1, n}=b_{1, n}$ and $\phi_{2}=\frac{\pi}{2}$.

The modal projection procedure developed allows describing the response of the shell for each of the modes involved. For the driven mode and the companion mode, their time phase shift can be observed. If these two associated modes are in phase or in opposition of phase, there is no travelling wave effect. This is the case in Figure 13, which shows the projection of the Popov's shell. This result corresponds to the findings in [17], where no travelling waves have been observed when analysing Popov's shell under pulsating loading.

It is interesting to note, that for the case of Booton's shell under parametric excitation, energy exchanges between the driven mode and companion modes can be observed. In these analysis cases, a travelling wave can arise, as can be seen in Figures 14 and 15, where the phenomenon occurs in the last part of the 200 forcing periods $T$ of the integration period. In the analysis case $\Omega / \omega_{\text {lin }}=1.9188$ (Figure 16) a long duration integration in resonance condition was performed. In this last case the energy exchange between driven and companion mode occurs without the phase shift in time corresponding to a travelling wave. 


\begin{tabular}{|c|c|c|c|c|c|c|c|}
\hline \multicolumn{2}{|c|}{$\Omega / \omega_{\text {lin }}=1.9068$} & \multicolumn{2}{|c|}{$\Omega / \omega_{\text {lin }}=1.9335$} & \multicolumn{2}{|c|}{$\Omega / \omega_{\text {lin }}=1.9601$} & \multicolumn{2}{|c|}{$\Omega / \omega_{\text {lin }}=1.9868$} \\
\hline$A_{\max } / h$ & mode & $A_{\max } / h$ & mode & $A_{\max } / h$ & mode & $A_{\max } / h$ & mode \\
\hline 3.4633 & 1,5 & 3.2063 & 1,5 & 3.0450 & 1,5 & 2.8232 & 1,5 \\
\hline 0.7823 & 1,0 & 0.6695 & 1,0 & 0.6229 & 1,0 & 0.5495 & 1,5 \\
\hline 0.6709 & 1,5 & 0.6229 & 1,5 & 0.5924 & 1,5 & 0.5400 & 1,0 \\
\hline 0.4811 & 1,10 & 0.3715 & 1,10 & 0.3593 & 1,10 & 0.3061 & 1,10 \\
\hline 0.3576 & 3,5 & 0.2528 & 3,5 & 0.2408 & 3,5 & 0.1770 & 3,5 \\
\hline 0.2608 & 3,10 & 0.2439 & 3,10 & 0.2156 & 3,10 & 0.1274 & 1,10 \\
\hline 0.2015 & 3,0 & 0.1822 & 5,5 & 0.1349 & 3,0 & 0.1078 & 3,0 \\
\hline 0.1871 & 5,5 & 0.1783 & 5,10 & 0.1248 & 1,10 & 0.1060 & 3,10 \\
\hline 0.1660 & 1,10 & 0.1669 & 3,0 & 0.1158 & 5,5 & 0.1048 & 5,10 \\
\hline 0.1442 & 5,10 & 0.1283 & 1,10 & 0.1056 & 3,10 & 0.0913 & 3,15 \\
\hline 0.1282 & 3,10 & 0.1182 & 3,10 & 0.0948 & 5,10 & 0.0617 & 5,5 \\
\hline 0.1029 & 7,10 & 0.0956 & 3,15 & 0.0717 & 7,10 & 0.0544 & 3,10 \\
\hline 0.0921 & 5,0 & 0.0853 & 5,0 & 0.0658 & 5,0 & 0.0464 & 5,0 \\
\hline 0.0891 & 1,15 & 0.0799 & 5,10 & 0.0564 & 3,15 & 0.0459 & 3,5 \\
\hline 0.0859 & 3,15 & 0.0792 & 7,10 & 0.0465 & 3,5 & 0.0411 & 3,15 \\
\hline 0.0684 & 3,5 & 0.0705 & 1,15 & 0.0451 & 1,15 & 0.0341 & 7,10 \\
\hline 0.0641 & 5,10 & 0.0520 & 3,15 & 0.0426 & 5,15 & 0.0253 & 5,10 \\
\hline 0.0594 & 5,15 & 0.0487 & 3,5 & 0.0426 & 5,10 & 0.0242 & 5,15 \\
\hline 0.0473 & 1,15 & 0.0486 & 5,15 & 0.0324 & 7,10 & 0.0211 & 5,15 \\
\hline 0.0473 & 3,15 & 0.0402 & 1,6 & 0.0317 & 3,15 & 0.0210 & 7,10 \\
\hline 0.0455 & 7,10 & 0.0366 & 1,15 & 0.0300 & 5,15 & 0.0206 & 1,15 \\
\hline 0.0420 & 1,6 & 0.0356 & 7,10 & 0.0242 & 1,15 & 0.0164 & 1,6 \\
\hline 0.0404 & 5,15 & 0.0344 & 5,5 & 0.0224 & 5,5 & 0.0111 & 1,6 \\
\hline
\end{tabular}

Table 6. Principal modes participation under parametric excitation for zero preload, Bogdanovich's isotropic shell. 


\begin{tabular}{llll}
\hline Instance & Ref. [17] & Ref. [12] & Present work \\
\hline Type & Modal damping & Modal damping & Rayleigh damping \\
& (all modes) & $($ all modes $)$ & \\
\hline Value & $\zeta_{\mathrm{i}}=0.089$ & $\zeta_{\mathrm{i}}=0.089$ & $\alpha=267.85$ \\
& & & $\beta=0.0000296$ \\
\hline
\end{tabular}

Table 7. Damping values for Popov's shell.

\begin{tabular}{lllll}
\hline$\Omega / \omega_{\text {lin }}$ & Ref. [17] & $\begin{array}{l}\text { Ref. [17] } \\
\text { (in-plane } \\
\text { inertia)* }\end{array}$ & Ref. [12] & $\begin{array}{l}\text { Present } \\
\text { work }\end{array}$ \\
\hline 1.9 & 0.473 & 0.439 & 0.448 & 0.435 \\
\hline 2.0 & 0.434 & 0.400 & 0.416 & 0.401 \\
\hline 2.1 & 0.524 & 0.479 & 0.492 & 0.488
\end{tabular}

Table 8. Critical dynamic load results comparison. *The values in Ref. [17] denoted with "in-plane inertia" correspond to a semi-analytical analysis including the in-plane inertia of the fundamental axial mode. 

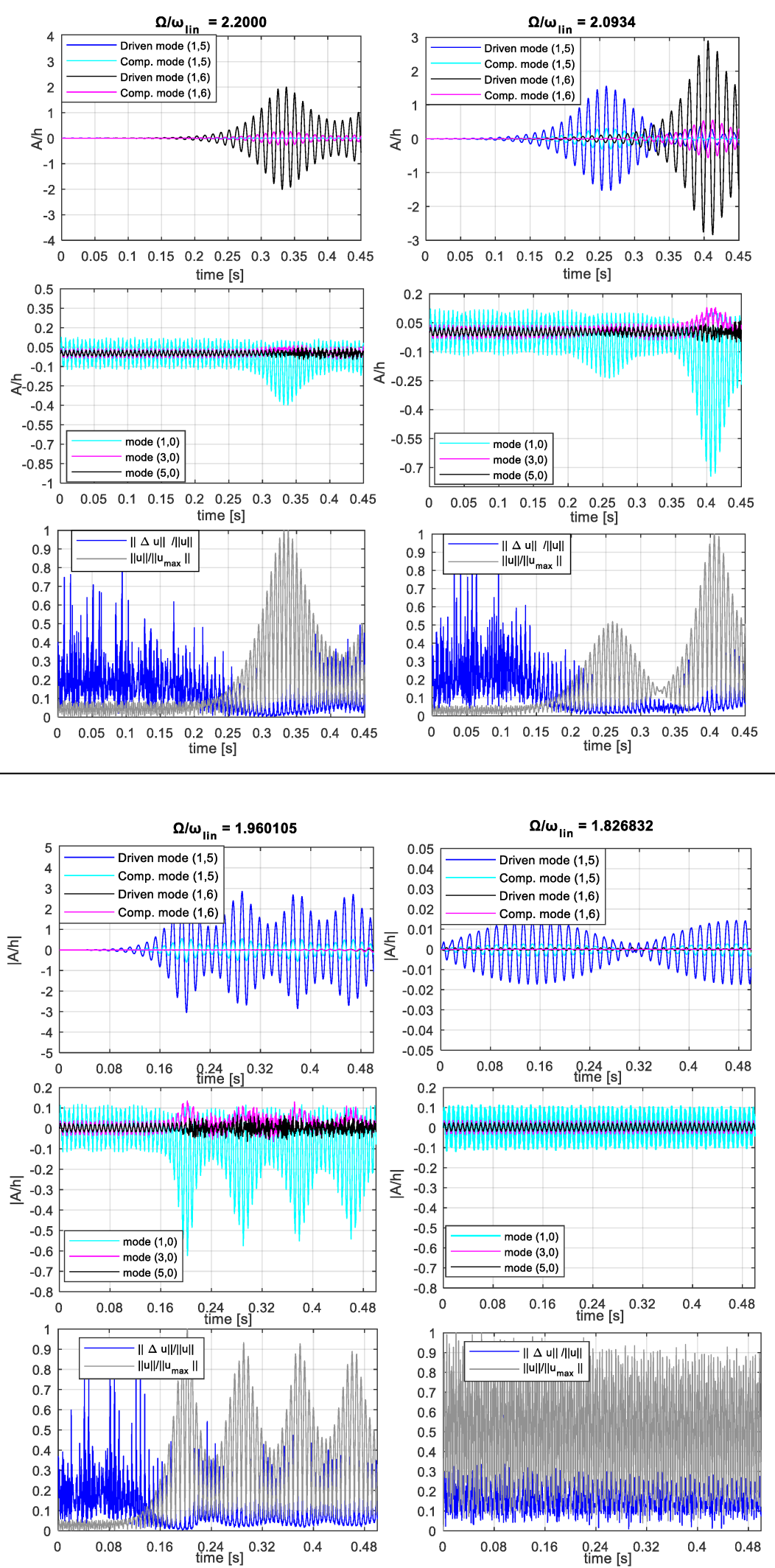

Figure 3. Time history of relevant modes for cylindrical shell under pulsating axial loading for zero preload through modal projection procedure for various excitation frequencies. A measure of the approximation error and the relative magnitude of the response are shown. Bogdanovich's isotropic shell. 


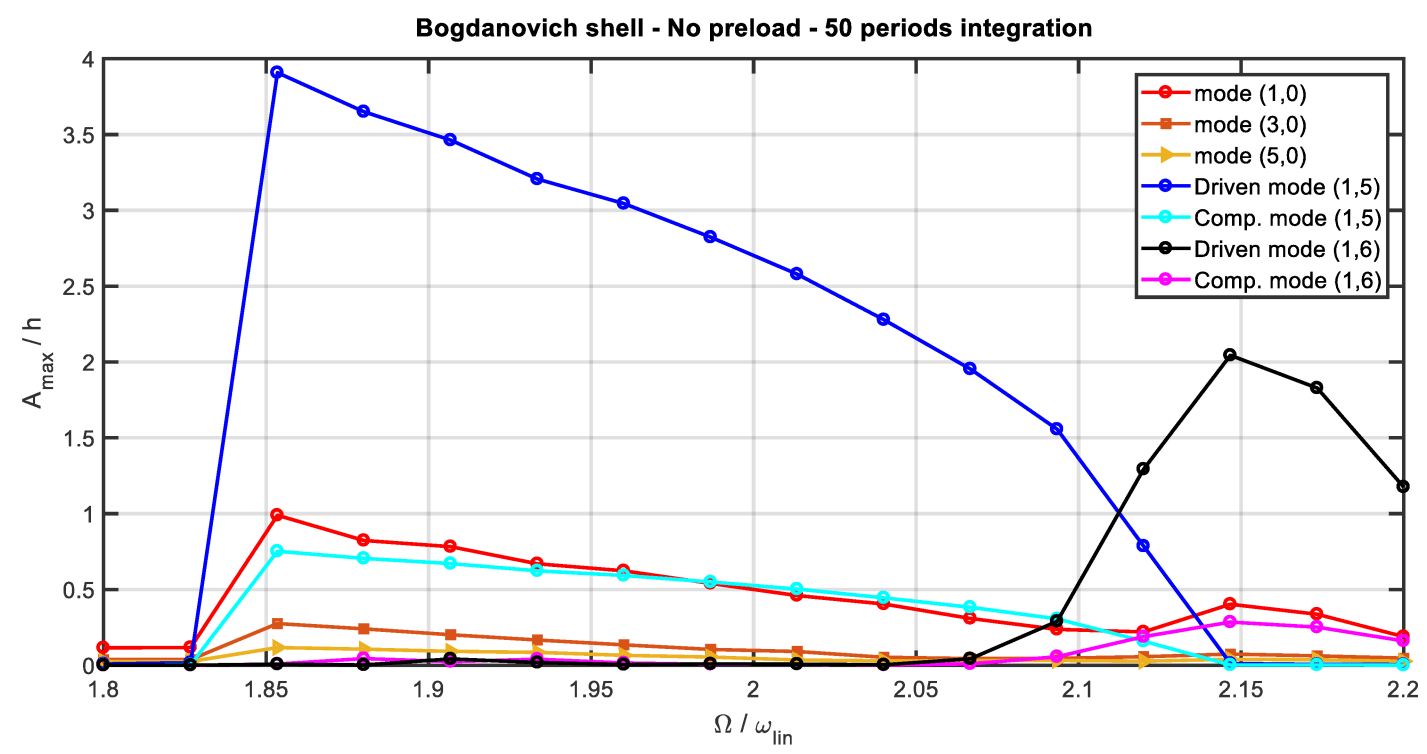

Figure 4. Frequency-response curves for cylindrical shell under pulsating axial loading for zero preload showing softening behaviour. Bogdanovich's isotropic shell.

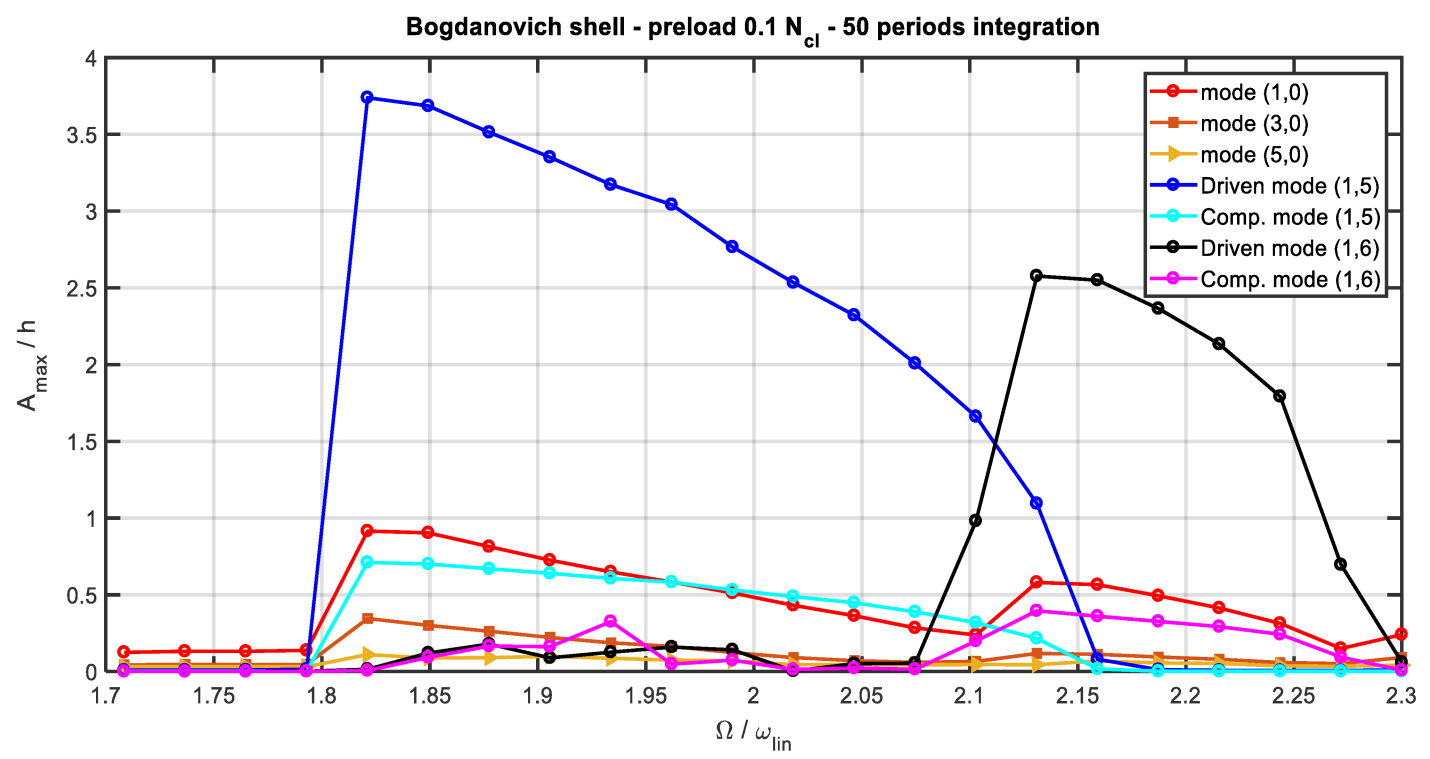

Figure 5. Frequency-response curves for cylindrical shell under pulsating axial loading for $0.1 \mathrm{~N}_{\mathrm{cl}}$ preload showing softening behaviour. Bogdanovich's isotropic shell. 


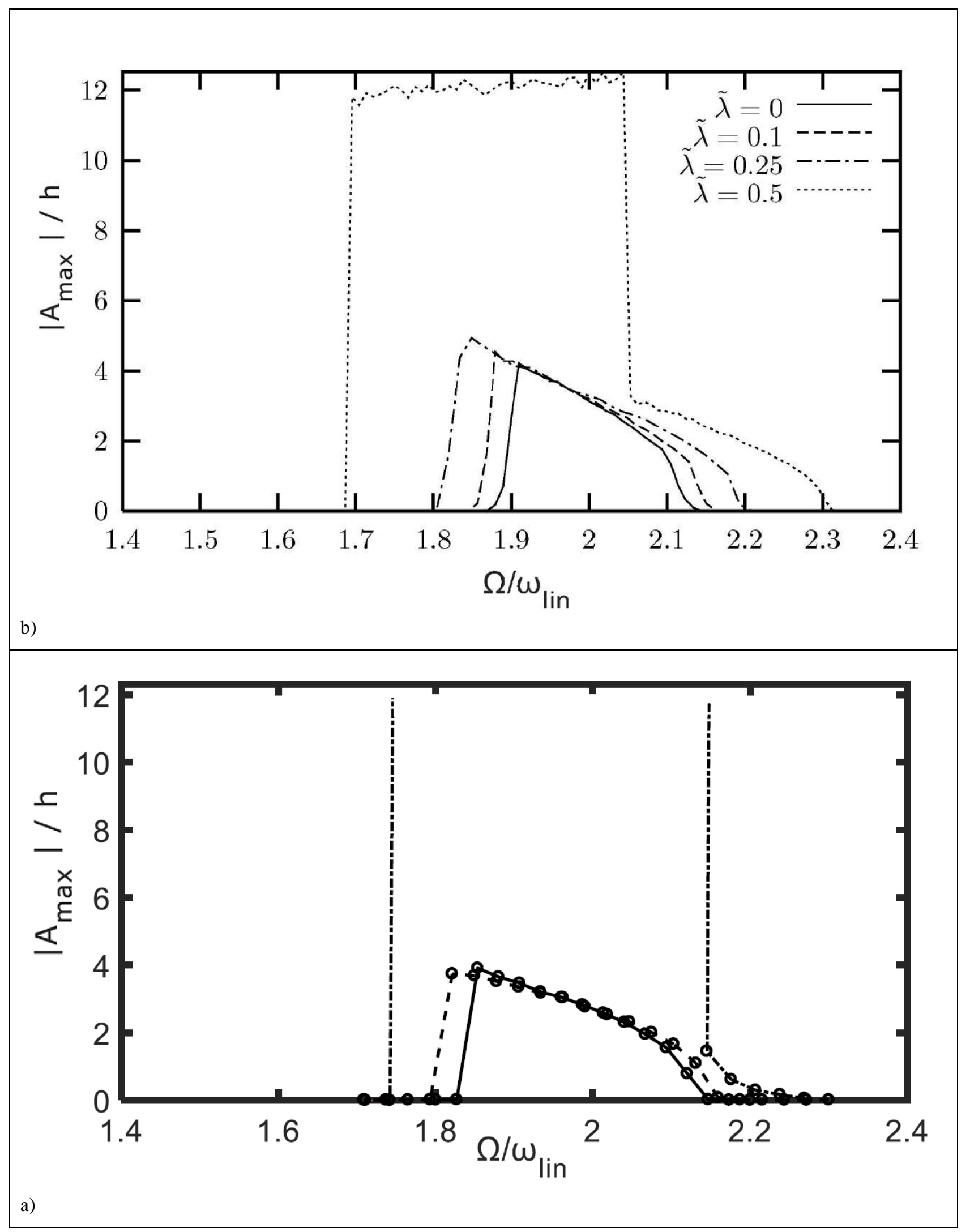

Figure 6. Frequency-response curves for cylindrical shell under pulsating axial loading of mode $(1,5)$ for the static preload levels $\tilde{\lambda}=\widetilde{\mathbf{N}}_{\mathbf{x}} / \mathbf{N}_{\mathbf{c l}}=0$ (solid line), 0.1 (dashed line), 0.25 (dash-dot line), 0.5 (dotted line). a) present FE result, b) semi-analytical result from Ref. [17]. $\omega_{\text {lin }}$ corresponds to the linear frequency of the respective pre-loaded shell. Bogdanovich's isotropic shell. 


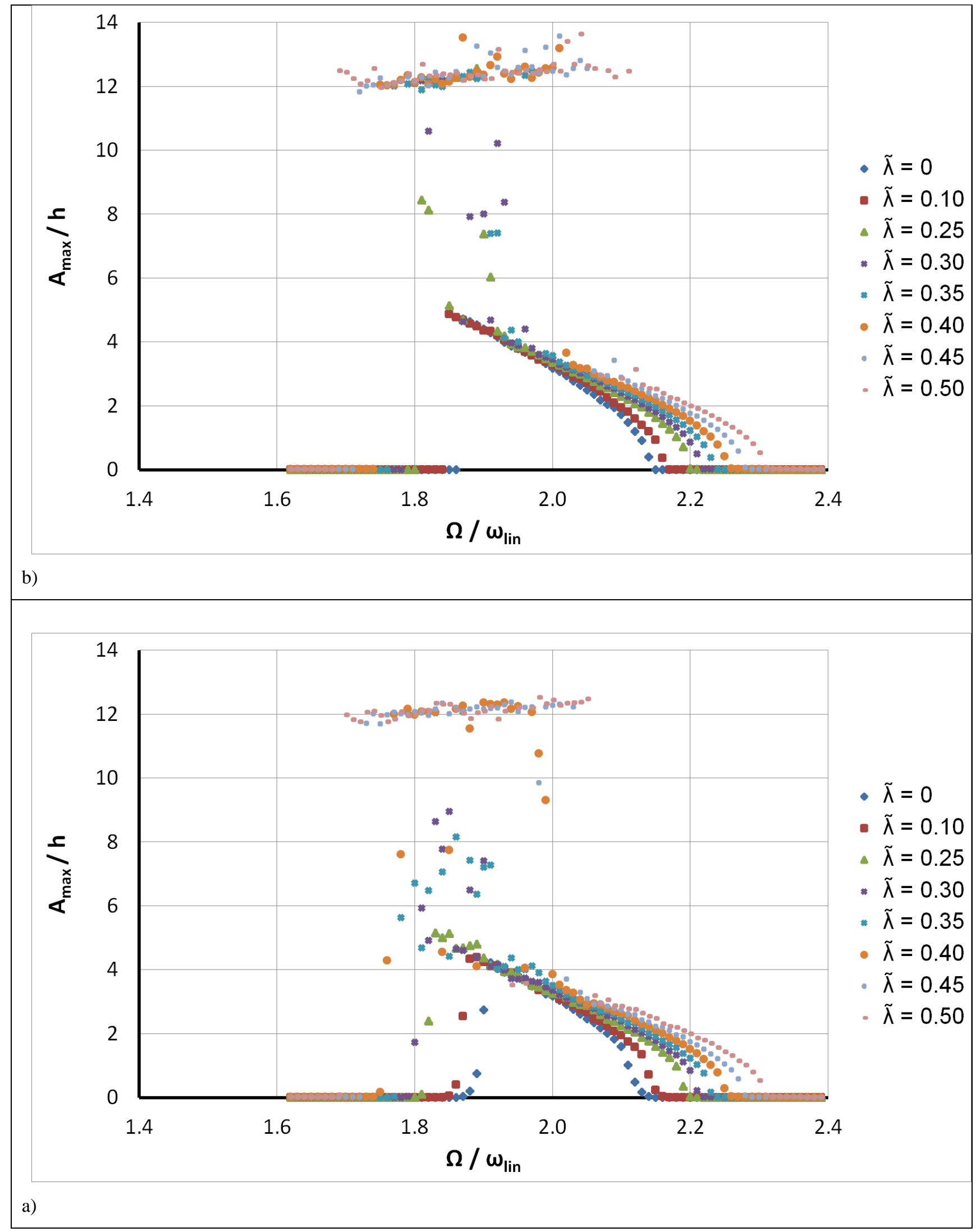

Figure 7. Frequency-response curves for cylindrical shell under pulsating axial loading of mode $(1,5)$ for various static pre-load levels $\tilde{\boldsymbol{\lambda}}=\widetilde{\mathbf{N}}_{\mathbf{x}} / \mathbf{N}_{\mathbf{c l}}=0,0.10,0.25,0.30,0.35,0.40,0.45,0.50$ using semi-analytical model from Ref. [17], a) number of forcing periods $=50, \mathrm{~b}$ ) number of forcing periods $=400 . \omega_{\operatorname{lin}}$ corresponds to the linear frequency of the respective pre-loaded shell. Bogdanovich's isotropic shell. 


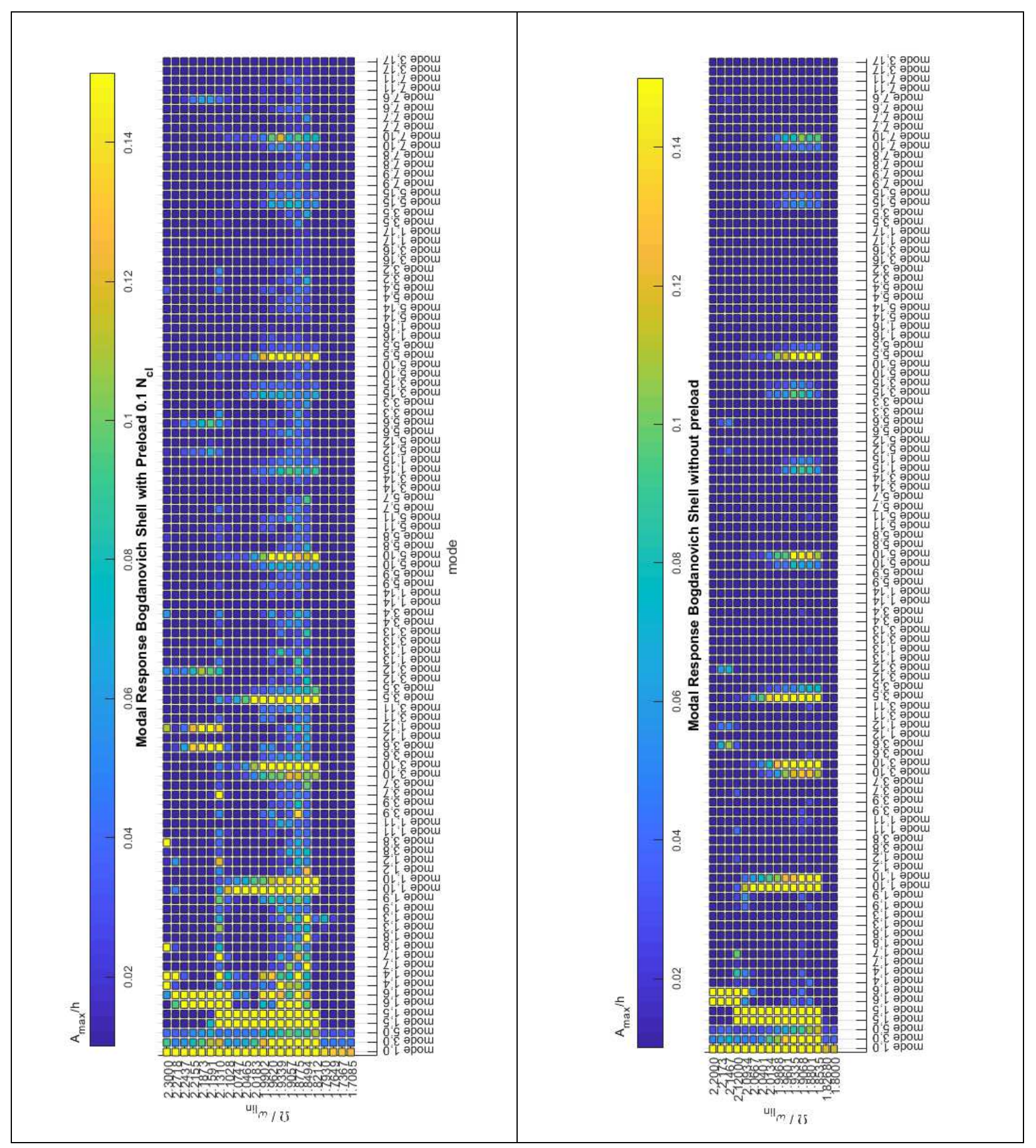

Figure 8. Waterfall chart for cylindrical shell under pulsating axial loading showing the contribution of various modes for varying excitation frequency. Bogdanovich's isotropic shell. 

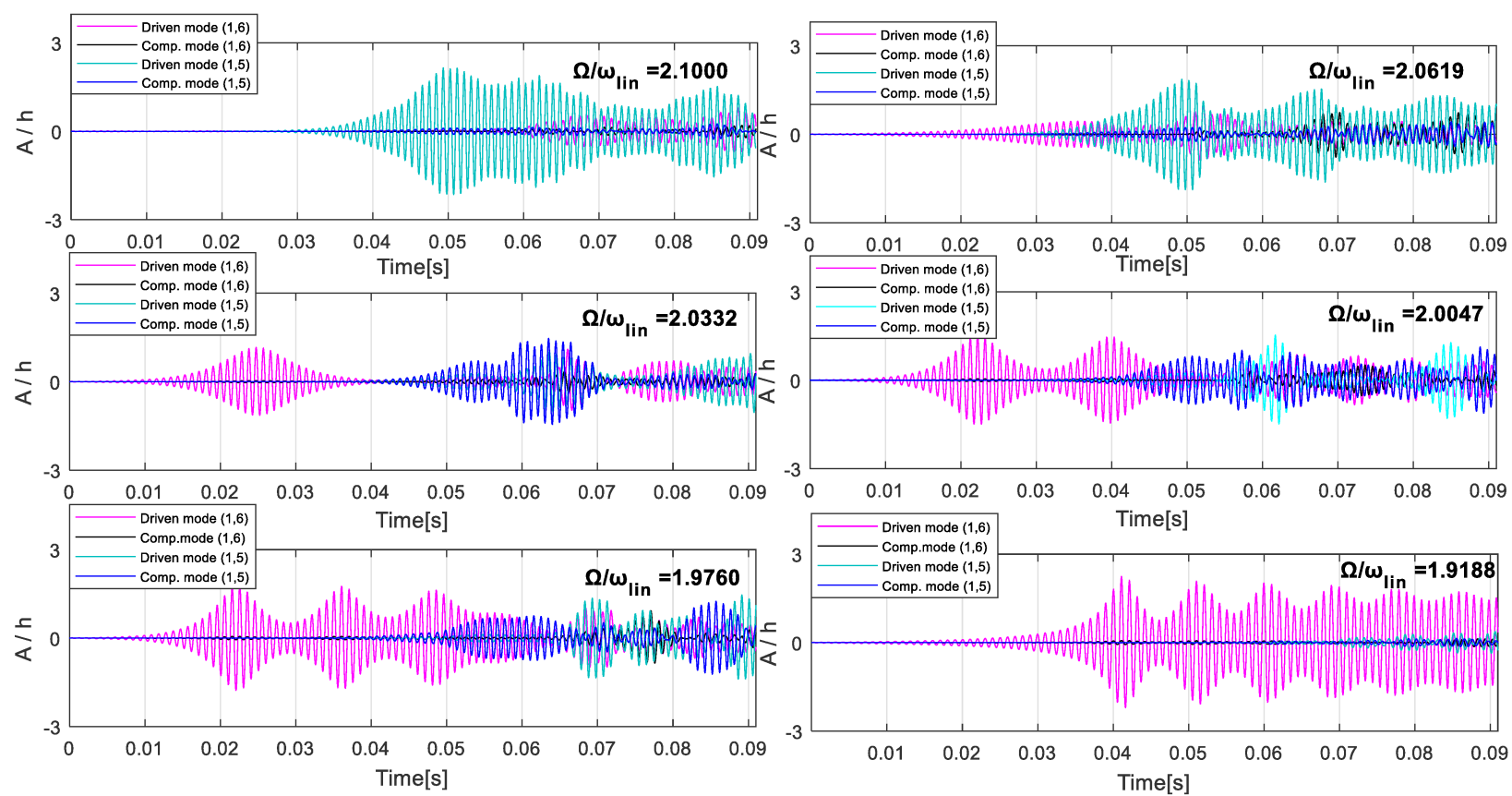

Figure 9. Time history of relevant modes for cylindrical shell under pulsating axial loading through modal projection procedure, mode $(1,5)$ - driven and companion mode, and mode $(1,6)$ - driven and companion mode. Booton's anisotropic shell. 


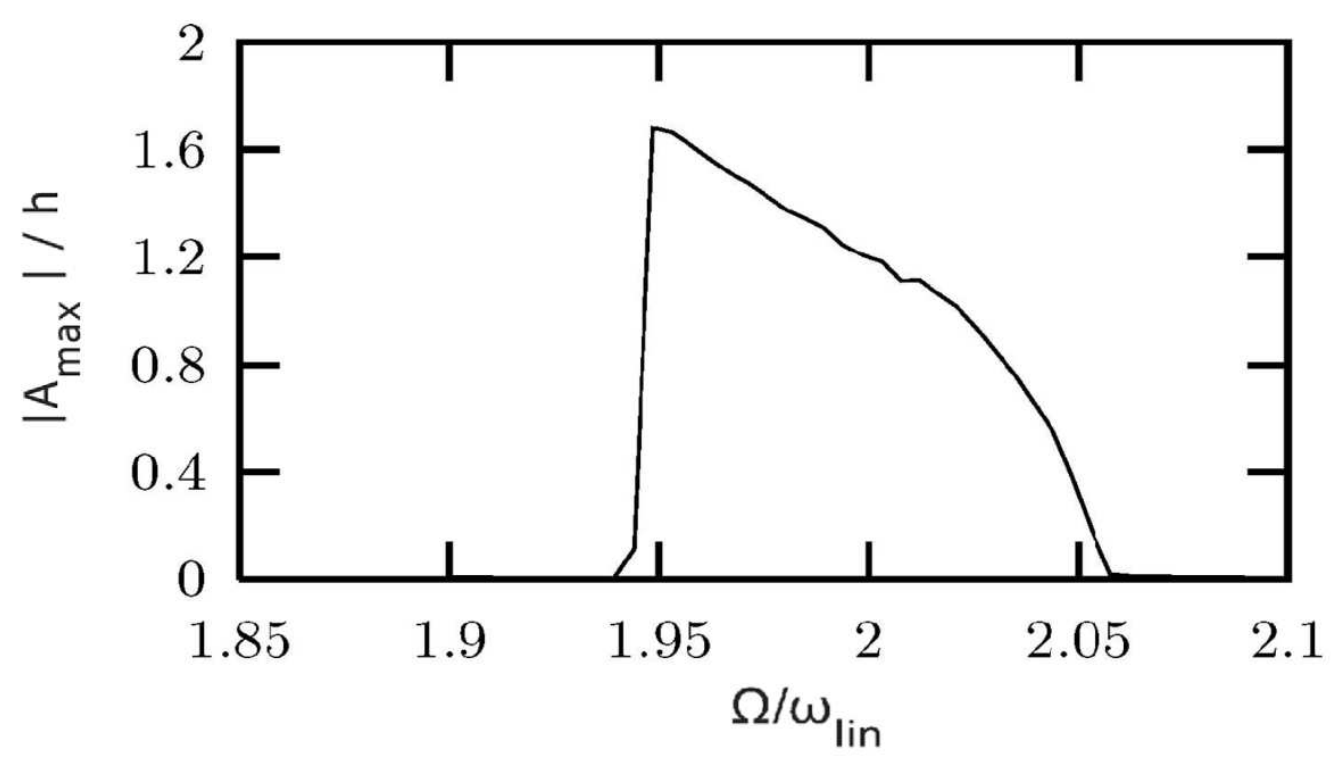

b)

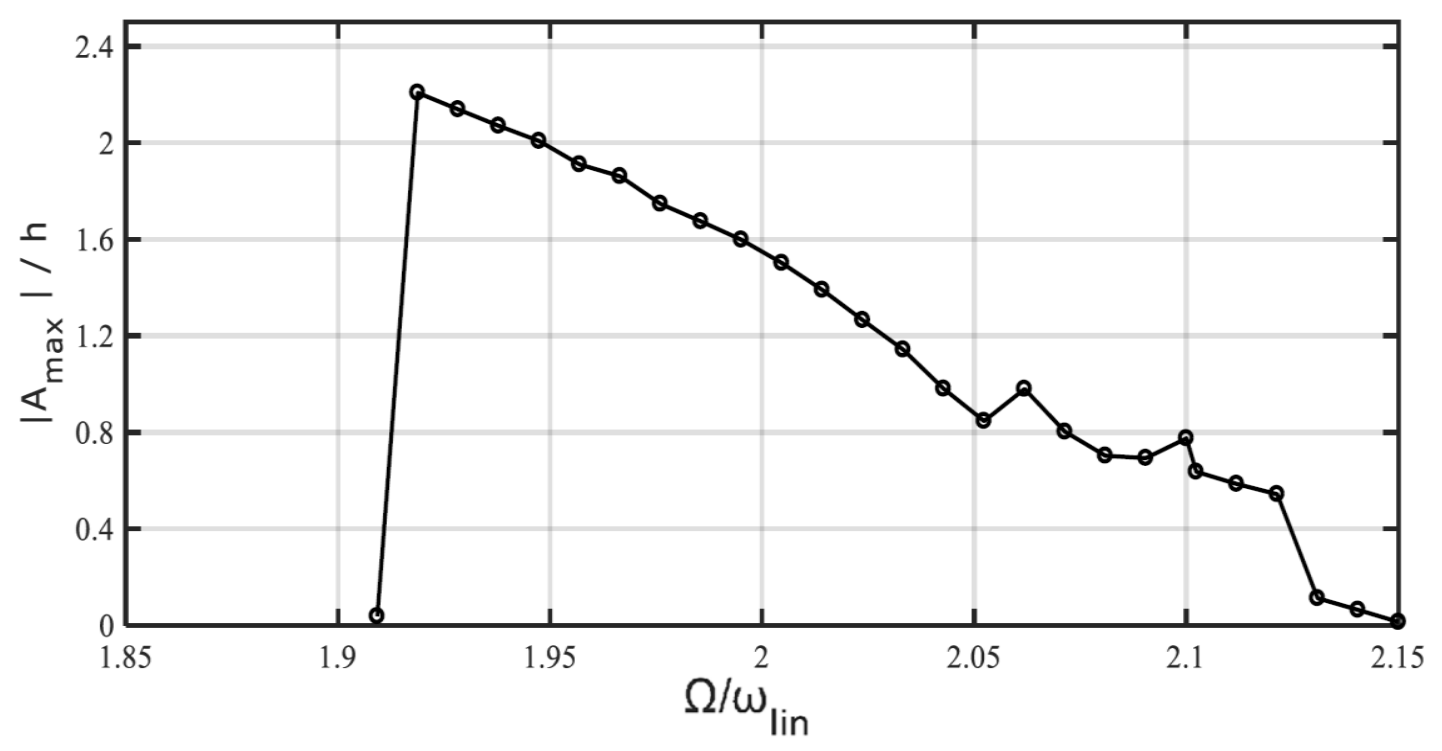

a)

Figure 10. Frequency-response curves for cylindrical shell under pulsating axial loading of mode $(1,6)$ for zero preload, a) present FE result, b) semi-analytical result from Ref. [17]. $\omega_{\text {lin }}$ corresponds to the linear frequency of the shell. Booton's anisotropic shell. 


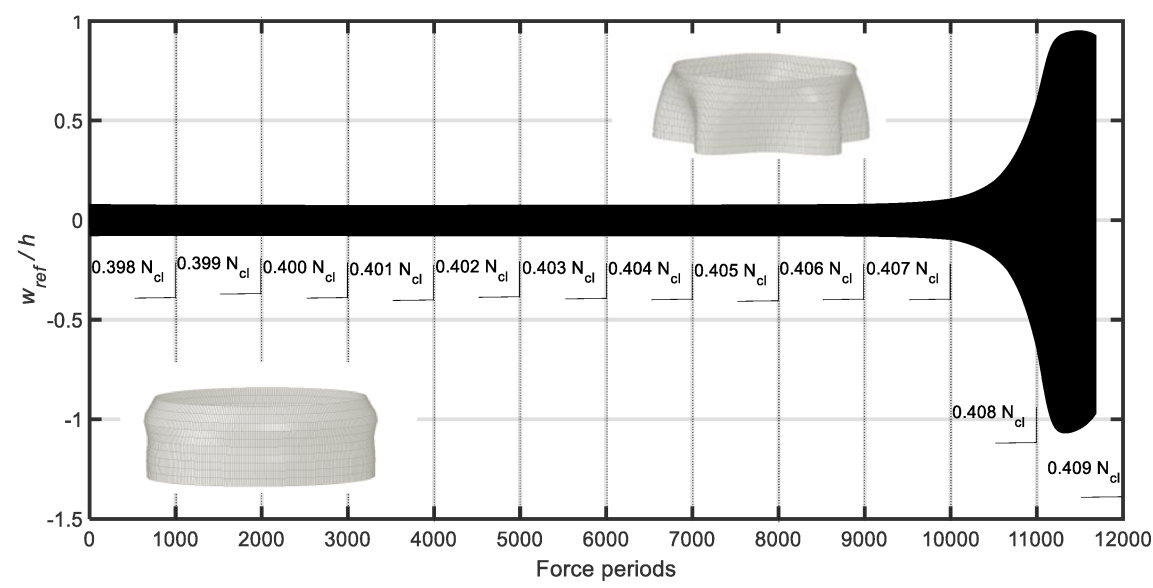

Figure 11. Time history and corresponding deformed shape for cylindrical shell under pulsating axial loading for $\Omega / \omega_{\text {lin }}$ $=2.0$ for increasing load amplitude. Radial displacement of a reference node at a maximum of the driven mode.

Popov's isotropic shell.
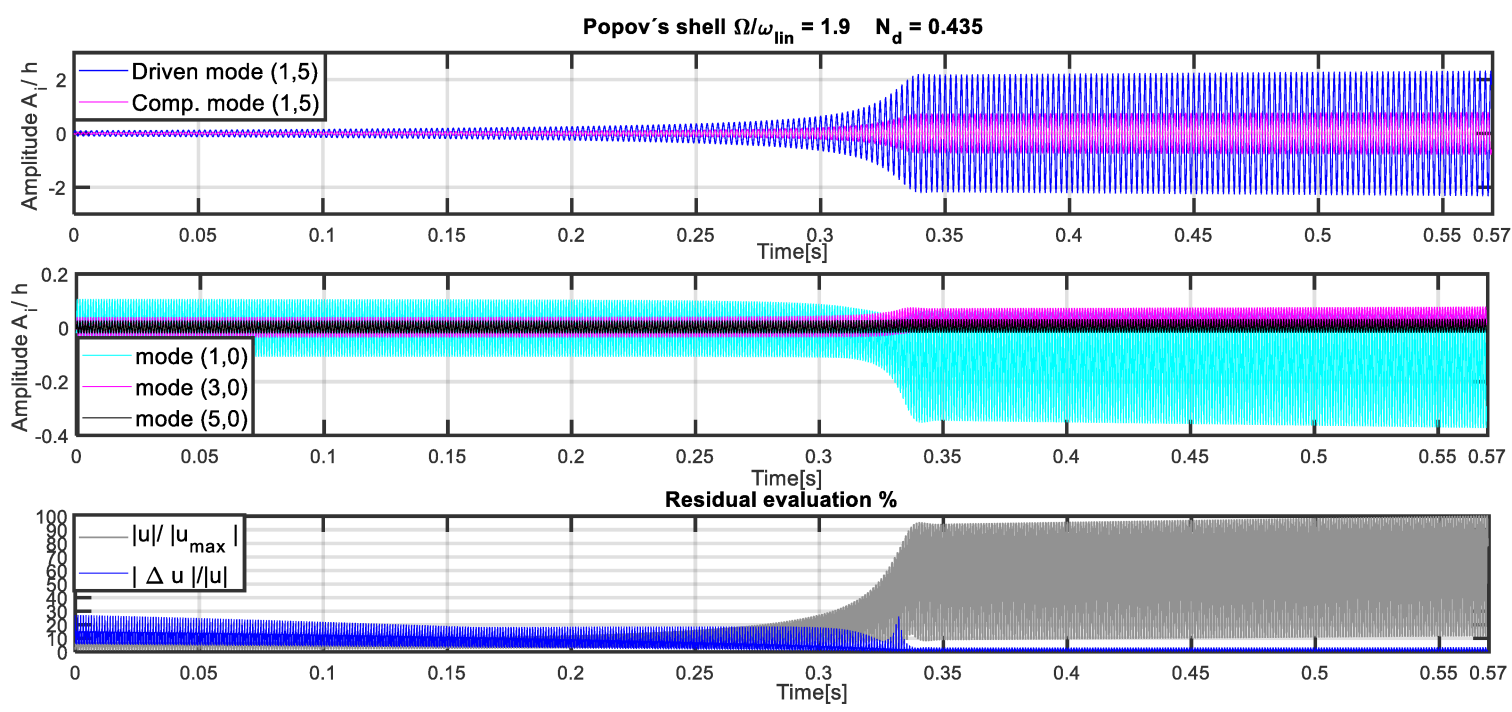

Figure 12. Time history of relevant modes for cylindrical shell under pulsating axial loading through modal projection procedure, showing occurrence of instability. Driven and companion modes and axisymmetric modes. Popov's isotropic shell. 


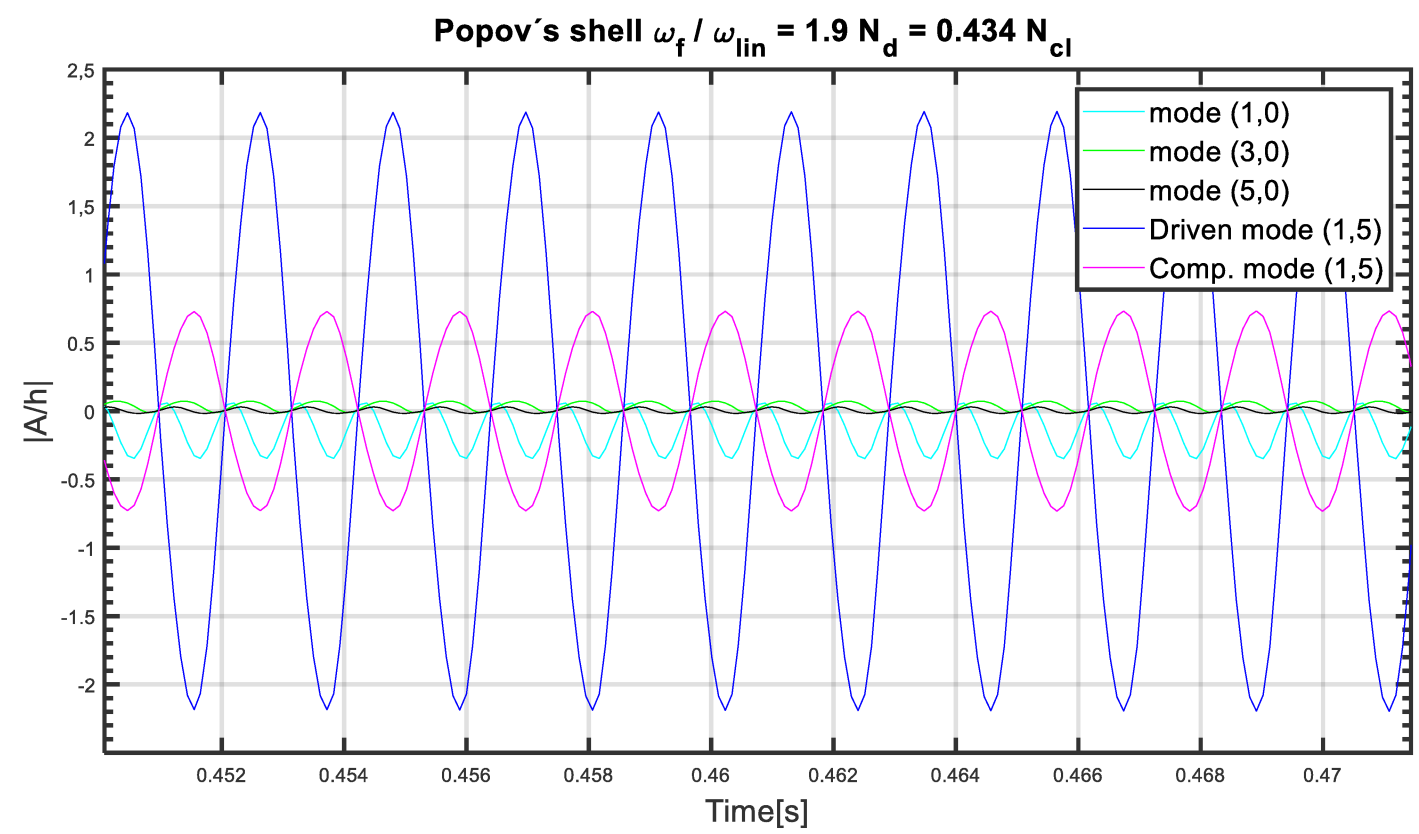

Figure 13. Time history of relevant modes for cylindrical shell under pulsating axial loading near parametric resonance through modal projection procedure. Driven mode and companion mode. A travelling wave phenomenon is not seen to occur. Popov's isotropic shell.

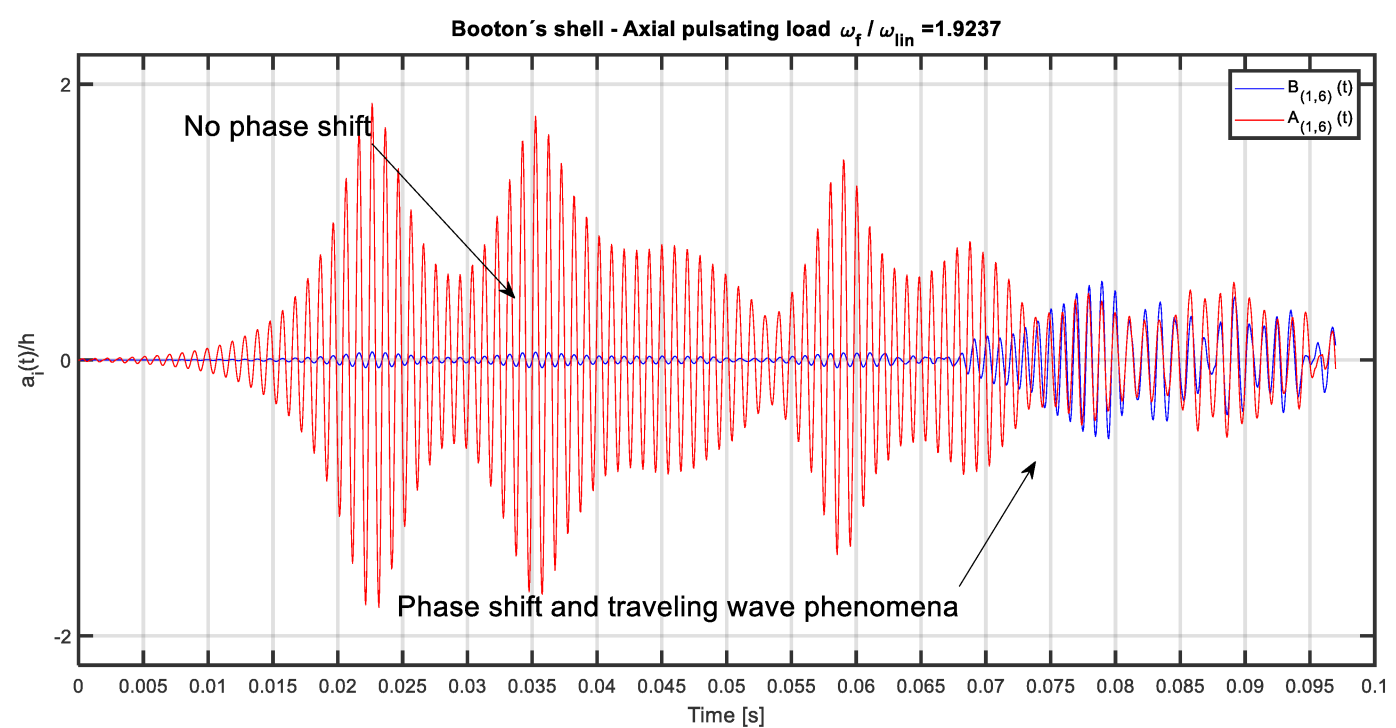

Figure 14. Time history of relevant modes for cylindrical shell under pulsating axial loading near parametric resonance through modal projection procedure showing, phase shift and travelling wave phenomenon. Driven mode and companion mode. $\Omega / \omega_{\text {lin }}=1.9237$. Booton's anisotropic shell. 


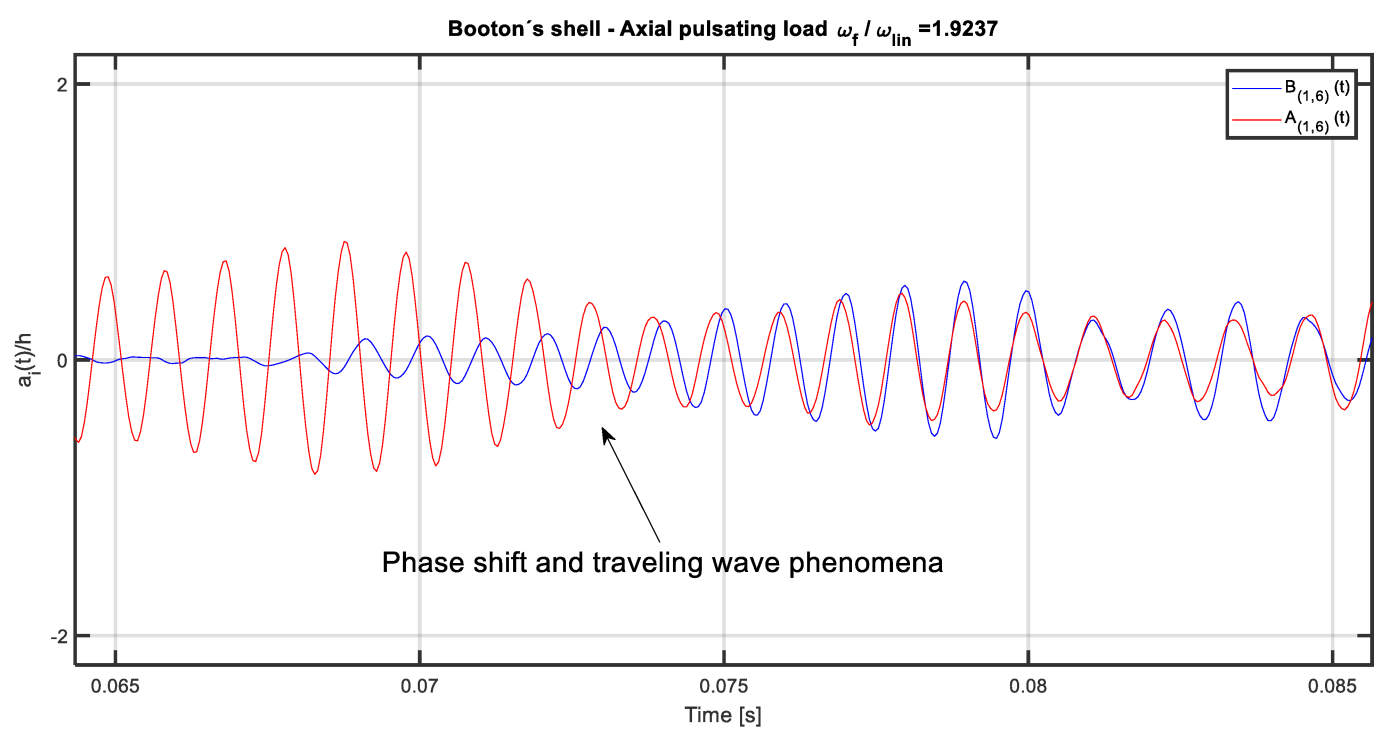

Figure 15. Detail of the time history of relevant modes for cylindrical shell under pulsating axial loading near parametric resonance through modal projection procedure, showing phase shift and travelling wave phenomenon.

Driven mode and companion mode. $\Omega / \omega_{\text {lin }}=1.9237$. Booton's anisotropic shell.

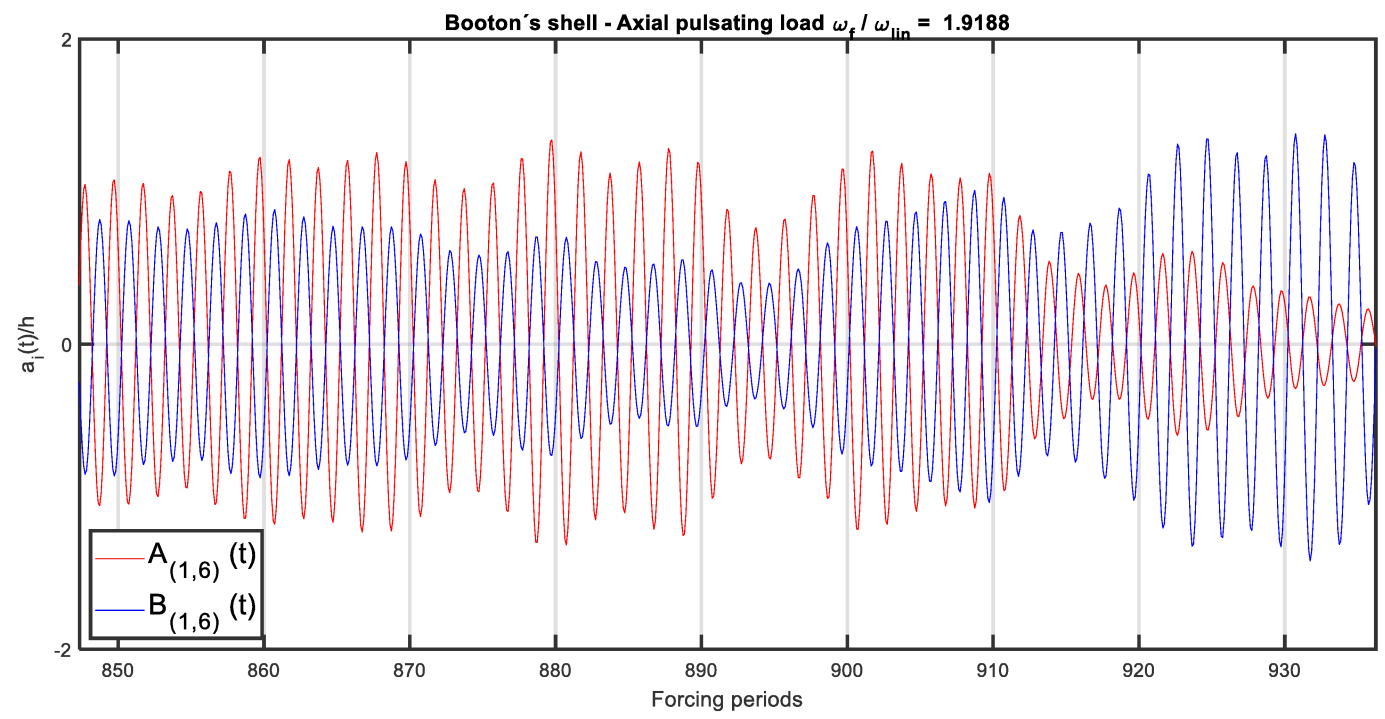

Figure 16. Long duration time history of relevant modes for cylindrical shell under pulsating axial loading near parametric resonance through modal projection procedure, showing energy exchange between driven mode and companion mode. $\Omega / \omega_{\text {lin }}=1.9188$. A travelling wave phenomenon is not seen to occur. Booton's anisotropic shell. 


\section{Concluding remarks}

In the present work dynamic stability investigations of isotropic and composite cylindrical shells under pulsating axial loading, in earlier work mainly investigated via semi-analytical procedures, have been carried out employing Finite Element analysis using numerical time integration. A combination of these two complementary approaches (Finite Element analysis and semi-analytical approach) is required to obtain reliable and accurate results in the complex and time-consuming nonlinear dynamic stability analysis.

The modal projection procedure for Finite Element analysis developed allows extracting modal amplitudes which can be directly compared with the corresponding modal amplitudes in the semianalytical approaches used in Refs. [12] and [17], so that a very good picture of the complex nonlinear behaviour occurring in the case of parametric excitation of cylindrical shells under axial loading can be obtained.

Critical dynamic loads and frequency-response were shown to be generally in good qualitative agreement with the findings presented in Ref. [17]. The present Finite Element approach, when compared with the simplified dynamic response analysis using a very limited number of assumed modes presented in Ref. [17], makes an improvement of the accuracy of the dynamic stability analysis of cylindrical shells possible. The analysis of the modal amplitude via the modal projection procedure developed gives the opportunity to study the interactions between the contributing modes and to interpret interesting phenomena such as the occurrence of travelling waves on the basis of a Finite Element analysis using numerical time integration.

\section{Acknowledgements}

The first two authors (Fabio Rizzetto and Eelco Jansen) would like to acknowledge the very helpful support of Dr. Cristian Gebhardt through sharing his expertise during the research stay of the first author at the institute headed by Prof. Raimund Rolfes, the Institute of Structural Analysis, Leibniz Universität Hannover. 


\section{References}

[1] Hsu, C. On the parametric excitation and snap-through stability problems of shells. Thin-Shell Structures. Theory, Experiment and Design. Prentice-Hall, New Jersey (1974) 103-131.

[2] Yao, J.C. Dynamic stability of cylindrical shells under static and periodic axial and radial loads. AIAA Journal 1(6) (1963) 1391-1396.

[3] Yao, J.C. Nonlinear elastic buckling and parametric excitation of a cylinder under axial loads. ASME Journal of Applied Mechanics 32(1) (1965) 109-115.

[4] Vijayaraghavan, A., Evan-Iwanowski, R. M. Parametric instability of circular cylindrical shells. ASME Journal of Applied Mechanics 34 (1967) 985-990.

[5] Nagai, K., Yamaki, N. Dynamic stability of circular cylindrical shells under periodic compressive forces. Journal of Sound and Vibration 58(3) (1978) 425-441.

[6] Argento, A. Dynamic stability of a composite circular cylindrical shell subjected to combined axial and torsional loading. Journal of Composite Materials 27 (1993) 1722-1738.

[7] Argento, A., Scott, R. Dynamic instability of layered anisotropic circular cylindrical shells: Part I. Theoretical developments. Journal of Sound and Vibration 162 (1993) 311-322.

[8] Argento, A., Scott, R. Dynamic instability of layered anisotropic circular cylindrical shells: Part II. Numerical results. Journal of Sound and Vibration 162 (1993) 323-332.

[9] Popov, A., Thompson, J., McRobie, F. Low-dimensional models of shell vibrations. Journal of Sound and Vibration 209 (1998) 163-186.

[10] Gonçalves, P.B., Del Prado, Z.G. Nonlinear oscillations and Stability of Parametrically Excited Cylindrical Shells. Meccanica 37 (2002) 569-597.

[11] Amabili, M. Nonlinear Vibrations and Stability of Shells and Plates. Cambridge University Press, New York, USA, 2008.

[12] Pellicano, F., Amabili, M. Stability and vibration of empty and fluid filled cylindrical shell under static and periodic axial loads. Journal of Solids and Structures 40 (2003) 3229-3251.

[13] Strozzi, M., Pellicano, F. Nonlinear vibrations of functionally graded cylindrical shells. ThinWalled Structures 67 (2013) 63-77.

[14] Pellicano, F., Amabili, M., Païdoussis, M.P. Effect of geometry on the non-linear vibration of circular cylindrical shells. International Journal of Non-Linear Mechanics 37 (2002) 1181-1198.

[15] Catellani, G., Pellicano, F., Dall'Asta, D., Amabili, M. Parametric instability of a circular cylindrical shell with geometric imperfections. Computers and Structures 82 (2004) 2635-2645.

[16] Jansen, E.L. Non-stationary flexural vibration behaviour of a cylindrical shell. International Journal of Non-Linear Mechanics 37 (2002) 937-949. 
[17] Jansen, E.L. Dynamic stability problems of anisotropic cylindrical shells via a simplified analysis. Nonlinear Dynamics 39 (2005) 349-367.

[18] Rahman, T., Jansen, E.L., Tiso, P. A Finite Element-Based perturbation method for nonlinear free vibration analysis of composite cylindrical shells. International Journal of Structural Stability and Dynamics 11(4) (2011) 717-734.

[19] Rahman, T., Jansen, E.L., Gürdal, Z. Dynamic buckling analysis of composite cylindrical shells using a finite element based perturbation method. Nonlinear Dynamics 66 (2011) 389-401.

[20] Saigal, S., Yang, T., Kapania, R. Dynamic buckling of imperfection sensitive shell structures. Journal of Aircraft 24 (1987) 718-724.

[21] Yaffe, R., Abramovich, H. Dynamic buckling of cylindrical stringer stiffened shells. Computers and Structures 81 (2003) 1031-1039.

[22] Bisagni, C. Dynamic buckling of fiber composite shells under impulsive axial compression. Thin-Walled Structures 43 (2005) 499-514.

[23] Ganapathi, M., Patel, B., Sambandam, C. Parametric dynamic instability analysis of laminated composite conical shells. Journal of Reinforced Plastics and Composites 18(14) (1999) 1336-1346. [24] Dey, T., Ramachandra, L.S. Dynamic stability of simply supported composite cylindrical shells under partial axial loading. Journal of Sound and Vibration 353 (2015) 272-291.

[25] Dassault Systèmes. Abaqus Analysis User's Guide. Providence, Rhode Island, USA (2016).

[26] Popov, A. Parametric resonance in cylindrical shells: a case of study in the nonlinear vibration of structural shells. Engineering Structures 25 (2003) 789-799.

[27] Bogdanovich, A.E. Non-Linear Dynamic Problems for Composite Cylindrical Shells. Elsevier Applied Science, London (1993).

[28] Booton, M., Tennyson, R.C. Buckling of Imperfect Anisotropic Circular Cylinders under Combined Loading. AIAA Journal 17(3) (1979) 278-287.

[29] Amabili, M., A. Sarkar, A., Païdoussis, M.P. Reduced-order models for nonlinear vibrations of cylindrical shells via the proper orthogonal decomposition method, Journal of Fluids and Structures 18 (2003) 227-250.

[30] Amabili, M., Touzé, C. Reduced-order models for nonlinear vibrations of fluid-filled circular cylindrical shells: Comparison of POD and asymptotic nonlinear normal modes methods, Journal of Fluids and Structures 23 (2007) 885-903.

[31] Gonçalves, P.B., Silva, F.M.A., Del Prado, Z.J.G.N. Low-dimensional models for the nonlinear vibration analysis of cylindrical shells based on a perturbation procedure and proper orthogonal decomposition, Journal of Sound and Vibration 315 (2008) 641-663. 